\title{
Charm-quark fragmentation fractions and production cross section at midrapidity in pp collisions at the LHC
}

\author{
S. Acharya et al. \\ (ALICE Collaboration)
}

(Received 2 August 2021; accepted 16 December 2021; published 12 January 2022)

\begin{abstract}
Recent $p_{\mathrm{T}}$-integrated cross-section measurements of the ground-state charm mesons and baryons, $\mathrm{D}^{0}$, $\mathrm{D}^{+}, \mathrm{D}_{\mathrm{s}}^{+}, \Lambda_{\mathrm{c}}^{+}$, and $\Xi_{\mathrm{c}}^{0}$, are used to evaluate the charm fragmentation fractions and production cross section per unit of rapidity at midrapidity $(|y|<0.5)$, in pp collisions at $\sqrt{s}=5.02 \mathrm{TeV}$ at the LHC. The latter is $\mathrm{d} \sigma^{\mathrm{c} \overline{\mathrm{c}}} /\left.\mathrm{d} y\right|_{|y|<0.5}=1165 \pm 44(\mathrm{stat})_{-101}^{+134}$ (syst) $\mu \mathrm{b}$. These measurements were obtained for the first time in hadronic collisions at the LHC, including the charm baryon states, recently measured by ALICE at midrapidity. The charm fragmentation fractions differ significantly from the values measured in $\mathrm{e}^{+} \mathrm{e}^{-}$and ep collisions, providing evidence of the dependence of the parton-to-hadron fragmentation fractions on the collision system, indicating that the assumption of their universality is not supported by the measured cross sections. An increase of a factor of about 3.3 for the fragmentation fraction for the $\Lambda_{c}^{+}$with a significance of $5 \sigma$ between the values obtained in pp collisions and those obtained in $\mathrm{e}^{+} \mathrm{e}^{-}$(ep) collisions is reported. The fragmentation fraction for the $\Xi_{\mathrm{c}}^{0}$ was obtained for the first time in any collision system. The measured fragmentation fractions were used to update the cc cross sections per unit of rapidity at $|y|<0.5$ at $\sqrt{s}=2.76$ and $7 \mathrm{TeV}$, which are about $40 \%$ higher than the previously published results. The data were compared with perturbative-QCD calculations and lie at the upper edge of the theoretical bands.
\end{abstract}

DOI: 10.1103/PhysRevD.105.L011103

The study of heavy-flavor hadron production in protonproton ( $\mathrm{pp}$ ) collisions provides an important test for quantum chromodynamics (QCD) calculations. The transverse-momentum $\left(p_{\mathrm{T}}\right)$ differential cross sections of charm mesons measured in pp collisions by the ALICE [1-5], ATLAS [6], CMS [7], and LHCb [8-10] experiments at the LHC and the STAR [11] experiment at RHIC, as well as in $\mathrm{p} \overline{\mathrm{p}}$ collisions by the CDF [12] experiment at the Tevatron, are described within uncertainties by perturbative-QCD (pQCD) calculations having next-to-leading order (NLO) accuracy with all-order resummation of next-to-leading logarithms, such as FONLL [13-15] and NLL [16-20]. These calculations are based on the factorization theorem, according to which the $p_{\mathrm{T}}$-differential cross sections are computed as the convolution of three terms: (i) the parton distribution functions (PDFs) of the incoming (anti)protons, (ii) the partonic cross section, calculated as a perturbative series in powers of the strong coupling constant $\alpha_{\mathrm{s}}$, and (iii) the fragmentation functions which describe the transition from charm quarks into charm

*Full author list given at the end of the article.

Published by the American Physical Society under the terms of the Creative Commons Attribution 4.0 International license. Further distribution of this work must maintain attribution to the author(s) and the published article's title, journal citation, and DOI. hadrons. The latter, in these calculations, are typically parametrized from measurements performed in $\mathrm{e}^{+} \mathrm{e}^{-}$or ep collisions [21], under the assumption that the hadronization of charm quarks into charm hadrons is a universal process independent of the colliding systems. Accordingly, measurements of charm mesons were exploited in the past to derive a measurement of the charm production cross section at hadron colliders, by scaling the production cross section of the D mesons with the corresponding charmquark fragmentation fraction $(\mathrm{FF}), f(\mathrm{c} \rightarrow \mathrm{D})$, taken from $\mathrm{e}^{+} \mathrm{e}^{-}$collisions $[1,3,9-11,22]$.

Recent measurements of charm-baryon production at midrapidity in $\mathrm{pp}$ collisions showed an enhancement of the $\Lambda_{c}^{+} / D^{0}$ [23-26] and $\Xi_{c}^{+, 0} / D^{0}$ [27-29] ratios for $p_{\mathrm{T}}<6-8 \mathrm{GeV} / c$ with respect to the ones measured in $\mathrm{e}^{+} \mathrm{e}^{-}$collisions. These measurements suggest a significant difference of the fragmentation fractions of charm quarks into charm baryons in hadronic collisions at LHC energies compared to those measured in $\mathrm{e}^{+} \mathrm{e}^{-}$and ep collisions. These findings are similar to those obtained in the beauty sector by the CDF Collaboration at the Tevatron [30] and by the LHCb Collaboration at the LHC [31,32]. Several models based on different assumptions, like the inclusion of hadronization via coalescence [33,34], or considering a set of yet-unobserved higher-mass charm-baryon states [35], or including string formation beyond the leadingcolor approximation [36], have been proposed to explain 
the baryon enhancement. Updates of the fit to the measured fragmentation functions of $\mathrm{c} \rightarrow \Lambda_{\mathrm{c}}^{+}$in $\mathrm{e}^{+} \mathrm{e}^{-}$collisions were also performed $[37,38]$ without improving the agreement between data and model calculations. These observations required a new approach for evaluating the charm-quark production cross section at midrapidity and the charmquark fragmentation fractions based on the measurements of both charm mesons and baryons.

The measurements described above not only provide constraints to pQCD calculations but are also important as references for the investigation of the charm-quark interaction with the medium created in heavy-ion collisions. In particular, in the context of the heavy-ion program at the LHC, the cc̄ production cross section per nucleon-nucleon collision is a fundamental ingredient for the determination of the amount of charmonium production by (re)generation in the quark-gluon plasma (QGP) [35,39-41], a mechanism that is supported by $\mathrm{J} / \psi$ measurements in nucleusnucleus collisions at the LHC $[42,43]$.

In this paper, the charm fragmentation fractions and the charm production cross section per unit of rapidity at midrapidity $(|y|<0.5)$ in pp collisions at $\sqrt{s}=5.02 \mathrm{TeV}$ are reported. The results were obtained by considering the contribution based on the measurement of the ground-state charm hadrons $\mathrm{D}^{0}, \mathrm{D}^{+}, \mathrm{D}_{\mathrm{s}}^{+}, \Lambda_{\mathrm{c}}^{+}$, and $\Xi_{\mathrm{c}}^{0}$ by the ALICE Collaboration [5,24,28].

The ALICE experiment and its performance are presented in detail in $[44,45]$. The main detectors used for the measurements presented here are the inner tracking system, the time projection chamber, and the time-of-flight detector for vertexing, tracking, and particle identification purposes. The data from $\mathrm{pp}$ collisions at $\sqrt{s}=5.02 \mathrm{TeV}$ were collected during the 2017 run with a minimum bias trigger,

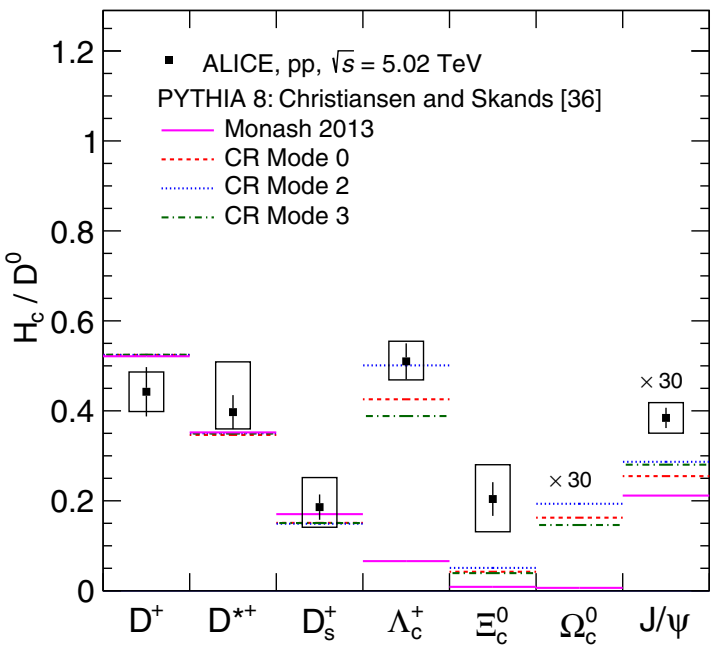

and they correspond to an integrated luminosity $L_{\text {int }}=(19.3 \pm 0.4) \mathrm{nb}^{-1}$ [46]. D mesons were reconstructed from their decays $\mathrm{D}^{0} \rightarrow \mathrm{K}^{-} \pi^{+}, \mathrm{D}^{+} \rightarrow \mathrm{K}^{-} \pi^{+} \pi^{+}$, $\mathrm{D}_{\mathrm{s}}^{+} \rightarrow \phi \pi^{+} \rightarrow \mathrm{K}^{-} \mathrm{K}^{+} \pi^{+}$, and $\mathrm{D}^{*+} \rightarrow \mathrm{D}^{0} \pi^{+}$, and charm baryons from their decays $\Lambda_{\mathrm{c}}^{+} \rightarrow \mathrm{pK}_{\mathrm{S}}^{0}, \Lambda_{\mathrm{c}}^{+} \rightarrow p K^{-} \pi^{+}$, and $\Xi_{\mathrm{c}}^{0} \rightarrow \Xi^{-} \mathrm{e}^{+} \nu_{\mathrm{e}}$. The charge conjugates are measured as well, and the results are averaged. The cross sections of $\mathrm{D}^{0}$ and $\mathrm{D}^{+}$mesons were measured down to $p_{\mathrm{T}}=0$ [5]. The cross sections for $\mathrm{D}^{*+}$ and $\mathrm{D}_{\mathrm{s}}^{+}$mesons were measured down to $p_{\mathrm{T}}=1 \mathrm{GeV} / c$, corresponding to about $80 \%$ of the integrated cross section [4]. The $\Lambda_{\mathrm{c}}^{+}$baryon cross section was measured down to $p_{\mathrm{T}}=1 \mathrm{GeV} / c$, corresponding to about $70 \%$ of the integrated cross sections $[24,25]$. The $\Xi_{\mathrm{c}}^{0}$ baryon was measured down to $p_{\mathrm{T}}=2 \mathrm{GeV} / c$, corresponding to about $40 \%$ of the integrated cross section [28]. The systematic uncertainties of the meson and baryon measurements include the following sources: (i) extraction of the raw yield, (ii) prompt fraction estimation, (iii) tracking and selection efficiency, (iv) particle identification efficiency, (v) sensitivity of the efficiencies to the hadron $p_{\mathrm{T}}$ shape generated in the simulation, and (vi) $p_{\mathrm{T}}$-extrapolation for the hadrons not measured down to $p_{\mathrm{T}}=0$. In addition, an overall normalization systematic uncertainty induced by the branching ratios (BR) [47] and the integrated luminosity [46] were considered.

Figure 1 shows the $p_{\mathrm{T}}$-integrated production cross sections per unit of rapidity of the various open- and hidden-charm meson $\left(\mathrm{D}^{+}, \mathrm{D}_{\mathrm{S}}^{+}, \mathrm{D}^{*+}\right.$, and $\left.\mathrm{J} / \psi\right)[4,5,48]$ and baryon $\left(\Lambda_{\mathrm{c}}^{+}\right.$and $\left.\Xi_{\mathrm{c}}^{0}\right)[24,25,28]$ species, obtained in pp collisions at $\sqrt{s}=5.02 \mathrm{TeV}$, as the average of particle and antiparticle, and normalized to the one of the $\mathrm{D}^{0}$ meson. When computing the ratios between the different hadron

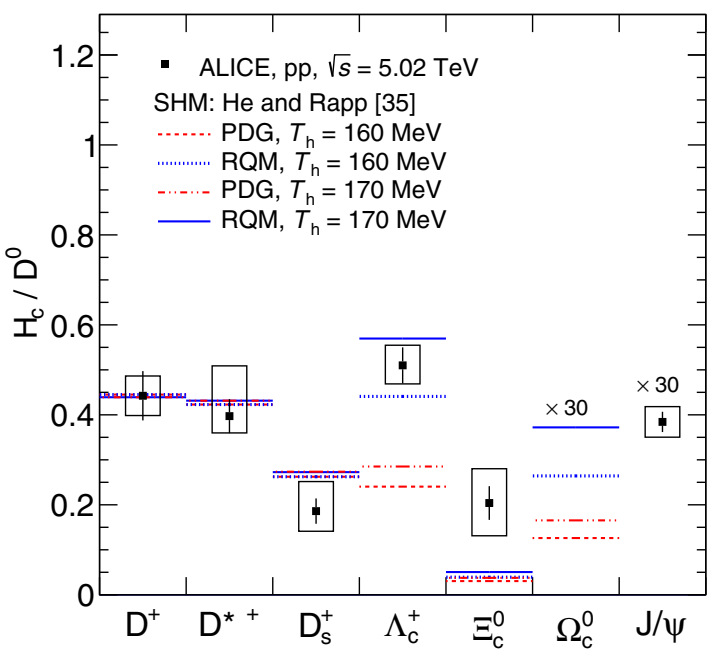

FIG. 1. Transverse-momentum integrated production cross sections of the various charm meson [4,5,48] and baryon [24,25,28] species per unit of rapidity at midrapidity normalized to that of the $\mathrm{D}^{0}$ meson measured in pp collisions at $\sqrt{s}=5.02 \mathrm{TeV}$. The measurements are compared with PYTHIA8 calculations [36,49] (left panel) and with results from a SHM [35] (right panel) (see text for details). For $\mathrm{J} / \psi$ the inclusive cross section was used. The $\mathrm{J} / \psi / \mathrm{D}^{0}$ ratio, as well as the model calculations for the $\Omega_{\mathrm{c}}^{0} / \mathrm{D}^{0}$ ratio, are multiplied by a factor 30 for visibility. 
species, systematic uncertainties due to tracking, the feeddown from beauty-hadron decays, the $p_{\mathrm{T}}$-extrapolation, and the luminosity were propagated as correlated. For the $\Xi_{\mathrm{c}}^{0}$ baryons, the additional contribution to the beauty feeddown systematic uncertainty due to the assumed $\Xi_{\mathrm{b}}^{0,-}$ baryon production relative to that of $\Lambda_{b}^{+}$baryons $[28,29]$ was considered as uncorrelated with the uncertainties related to the beauty feed-down subtraction for the other charm hadron species. In the $\mathrm{J} / \psi / \mathrm{D}^{0}$ ratio all the systematic uncertainties were propagated as uncorrelated, with the exception of the luminosity uncertainty. The treatment of the systematic uncertainties is also the same for the computation of the other quantities reported here.

In the left panel of Fig. 1 the experimental data are compared with results from the PYTHIA8 generator, using the Monash 2013 tune [49], and tunes that implement color reconnections (CR) beyond the leading-color approximation [36]. In the Monash 2013 tune, the parameters governing the heavy-quark fragmentation are tuned to measurements in $\mathrm{e}^{+} \mathrm{e}^{-}$collisions. The CR tunes introduce new color reconnection topologies, including junctions, that enhance the baryon production and, to a lesser extent, charmonia. The three considered tunes (Modes 0, 2, and 3) apply different constraints on the allowed string reconnections, taking into account causal connections of dipoles involved in a reconnection and time dilation effects caused by relative boosts between string pieces. While multiparton interactions (MPI) are observed in PYTHIA8 to significantly increase the charm quark production, a modification of the relative abundances of the charm hadron species, with the relative baryon enhancement, is observed only when the MPI are coupled to a color reconnection mode beyond the leading-color approximation [49]. It is observed that for the open charm meson ratios, the PYTHIA8 generator predictions with the different tunes are fairly similar and describe the measurements within uncertainty, except for the $\mathrm{D}^{+} / \mathrm{D}^{0}$ ratio, which is overestimated by about $15 \%$. However, this difference has a significance of only 1 standard deviation of the combined statistical and systematic uncertainties. Significant differences in the PYTHIA8 predictions are observed when comparing them with the measured baryon-to-meson ratios. The Monash 2013 tune is observed to underestimate the $\Lambda_{\mathrm{c}}^{+} / \mathrm{D}^{0}$ and $\Xi_{\mathrm{c}}^{0} / \mathrm{D}^{0}$ ratios by nearly $8 \sigma$ and $2.3 \sigma$, respectively. It is significantly different from all the CR tunes, which provide an increase of the baryon-to-meson ratio. Mode 2 is the PYTHIA8 tune describing the $\Lambda_{\mathrm{c}}^{+} / \mathrm{D}^{0}$ ratio; however, it still underestimates the $\Xi_{\mathrm{c}}^{0} / \mathrm{D}^{0}$ ratio by about $2 \sigma$. For the $\mathrm{J} / \psi / \mathrm{D}^{0}$ ratio the $\mathrm{CR}$ tunes provide a better description than the Monash 2013 tune. However, all PYTHIA8 tunes underestimate the measurement. In the simulations, as in the experimental measurement, the $\mathrm{J} / \psi$ cross section consists of the prompt and beauty feed-down contributions. The fraction of $\mathrm{J} / \psi$ from the decay of b-hadrons is about $15 \%$ for $p_{\mathrm{T}}^{\mathrm{J} / \psi}>$ $1.3 \mathrm{GeV} / c$ [50-52].
In the right panel of Fig. 1, the measurements are compared with two versions of a statistical hadronization model (SHM) [35]. One is based on the charm baryon states included by the Particle Data Group (PDG) [47], while the other version includes an augmented set of charm baryon states, given by predictions of the relativistic quark model (RQM) [53]. Both versions are reported for two different hadronization temperatures $\left(T_{\mathrm{h}}\right)$ [35]. The two $T_{\mathrm{h}}$ values of $160 \mathrm{MeV}$ and $170 \mathrm{MeV}$ used in the model are above the temperature of $156.5 \mathrm{MeV}$ reported from a fit to the lightflavor hadron yields in central $\mathrm{Pb}-\mathrm{Pb}$ collisions [54,55]. The implementation of the two hadronization temperatures leads only to small variations in the meson-to-meson ratios, while more significant changes are observed in the baryon sector. The charm mesons $\mathrm{D}^{0}, \mathrm{D}^{+}$, and $\mathrm{D}_{\mathrm{s}}^{+}$and baryons are dominantly populated by strong decays from higher-lying charm resonances. Therefore, changes due to an increased temperature on yield ratios relative to $\mathrm{D}^{0}$ are due to subtle effects. In particular, in the meson-to-meson ratios a weak sensitivity to temperature and no change due to the added baryons is visible. For the charm baryons, even with the standard PDG spectrum, there is a stronger sensitivity to a temperature increase (dashed and dash-dotted red lines in the right panel of Fig. 1). The additional baryon states almost double the fraction of the ground-state $\Lambda_{\mathrm{c}}^{+}$in the system relative to the PDG scenario, when a hadronization temperature of $170 \mathrm{MeV}$ is used, and the resulting $\Lambda_{\mathrm{c}}^{+} / \mathrm{D}^{0}$ ratio becomes comparable to the ALICE measurement [24]. A similar conclusion is drawn for the production cross section of $\Sigma_{\mathrm{c}}^{0,+,++}$ baryons in pp collisions at $\sqrt{s}=13 \mathrm{TeV}$ [56]. The $\Xi_{\mathrm{c}}^{0} / \mathrm{D}^{0}$ ratio is observed to increase by a factor 1.3 with respect to the PDG case. With this increase of the $\Xi_{c}^{0}$ yield, the model calculation is compatible with the measurement within $1.8 \sigma$. No model calculation is available for the $\mathrm{J} / \psi / \mathrm{D}^{0}$ ratio.

The cic production cross section per unit of rapidity at midrapidity $\left(\mathrm{d} \sigma^{c \bar{c}} /\left.\mathrm{d} y\right|_{|y|<0.5}\right)$ was calculated by summing the $p_{\mathrm{T}}$-integrated cross sections of all measured groundstate charm hadrons $\left(\mathrm{D}^{0}, \mathrm{D}^{+}, \mathrm{D}_{\mathrm{s}}^{+}, \Lambda_{\mathrm{c}}^{+}\right.$, and $\left.\Xi_{\mathrm{c}}^{0}\right)$. The contribution of the $\Xi_{\mathrm{c}}^{0}$ was multiplied by a factor of 2 , in order to account for the contribution of the $\Xi_{c}^{+}$. The production cross sections of the $\Xi_{c}^{0}$ and $\Xi_{c}^{+}$baryons were found to be compatible within experimental uncertainties in pp collisions at $\sqrt{s}=13 \mathrm{TeV}$ [29]. The contribution of $\mathrm{J} / \psi$ to the charm production cross section at midrapidity was considered negligible with respect to the other hadron species. Given the absence of measurements of $\Omega_{\mathrm{c}}^{0}$ baryon production at hadron colliders, an asymmetric systematic uncertainty was assigned assuming a contribution equal to the one of $\Xi_{\mathrm{c}}^{0}$ considering the prediction of the Catania model [34]. This uncertainty was summed in quadrature with the other extrapolation uncertainties. Two correction factors for the different shapes of the rapidity distributions

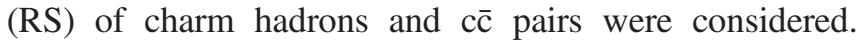


The first factor accounts for the different rapidity distributions of charm hadrons and single charm quarks, and it was evaluated to be unity in the relevant rapidity range based on FONLL calculations. A 2\% uncertainty on this factor was evaluated from the difference obtained with PYTHIA8. The second correction factor was computed as the ratio $\left(\mathrm{d} \sigma^{\mathrm{c} \overline{\mathrm{c}}} / \mathrm{d} y\right) /\left(\mathrm{d} \sigma^{\mathrm{c}} / \mathrm{d} y\right)$, which was estimated from NLO pQCD calculations (POWHEG [57]) to be 1.03 . A $3 \%$ uncertainty on this factor was estimated from the difference among the values obtained by varying the factorization and renormalization scales independently by a factor of 2 in the POWHEG calculation and using different sets of PDFs (CT10NLO [58], CT14NLO [59], CT18NNLO [60], CTEQ66 [61], and NNPDF31NNLO [62]). The resulting c c cross section per unit of rapidity at midrapidity is

$$
\begin{aligned}
\left.\frac{\mathrm{d} \sigma^{\mathrm{c} \overline{\mathrm{c}}}}{\mathrm{d} y}\right|_{|y|<0.5} ^{\mathrm{pp}, 5.02 \mathrm{TeV}}= & 1165 \pm 44(\text { stat })_{-67}^{+63}(\text { syst })_{-38}^{+98}(\text { extr }) \\
& \pm 43(\mathrm{BR}) \pm 42(\mathrm{RS}) \pm 24(\text { lumi }) \mu \mathrm{b} .
\end{aligned}
$$

The reported uncertainties in Eq. (1) named (extr) and (BR) refer to extrapolation uncertainties of the charmhadron cross sections not measured down to $p_{\mathrm{T}}=0$ and to the uncertainties of the branching ratios. The extrapolated fraction of the cross section is smaller than 20\%. More details on the extrapolation uncertainties are reported in $[5,25,28]$.

The charm fragmentation fractions, $f\left(\mathrm{c} \rightarrow \mathrm{H}_{\mathrm{c}}\right)$, which represent the probabilities of a $\mathrm{c}$ quark to hadronize into a given charm hadron, are listed in Table I. They were obtained by dividing the $p_{\mathrm{T}}$-integrated cross section of each measured hadron species by the sum of the cross sections of the different ground-state charm hadron species, considering twice the contribution of the $\Xi_{\mathrm{c}}^{0}$ baryon. An asymmetric uncertainty to account for the possible sizable contribution of $\Omega_{\mathrm{c}}^{0}$ was added as done for the evaluation of $\mathrm{d} \sigma^{\mathrm{c} \overline{\mathrm{c}}} / \mathrm{d} y$.

TABLE I. Charm-quark fragmentation fractions into charm hadrons, $f\left(\mathrm{c} \rightarrow \mathrm{H}_{\mathrm{c}}\right)$, determined from measurements in $\mathrm{pp}$ collisions at $\sqrt{s}=5.02 \mathrm{TeV}$. Statistical and systematic uncertainties are reported separately. To obtain the complete fragmentation of a c quark, an additional contribution equal to the one of the $\Xi_{\mathrm{c}}^{0}$ should be added to account for the $\Xi_{\mathrm{c}}^{+}$. The $f\left(\mathrm{c} \rightarrow \Lambda_{\mathrm{c}}^{+}\right)$ includes the feed-down from $\Sigma_{\mathrm{c}}^{0,+,++}$ baryons. The sum of the fragmentation fractions adds up to unity within uncertainties, not

\begin{tabular}{|c|c|}
\hline $\mathrm{H}_{\mathrm{c}}$ & $f\left(\mathrm{c} \rightarrow \mathrm{H}_{\mathrm{c}}\right)(\%)$ \\
\hline $\mathrm{D}^{0}$ & $39.1 \pm 1.7(\text { stat })_{-3.7}^{+2.5}($ syst $)$ \\
\hline $\mathrm{D}^{+}$ & $17.3 \pm 1.8(\text { stat })_{-2.1}^{+1.7}(\mathrm{syst})$ \\
\hline $\mathrm{D}_{\mathrm{s}}^{+}$ & $7.3 \pm 1.0(\text { stat })_{-1.1}^{+1.9}($ syst $)$ \\
\hline$\Lambda_{\mathrm{c}}^{+}$ & $20.4 \pm 1.3(\mathrm{stat})_{-2.2}^{+1.6}(\mathrm{syst})$ \\
\hline$\Xi_{\mathrm{c}}^{0}$ & $8.0 \pm 1.2(\text { stat })_{-2.4}^{+2.5}($ syst $)$ \\
\hline $\mathrm{D}^{*+}$ & $15.5 \pm 1.2(\mathrm{stat})_{-1.9}^{+4.1}(\mathrm{syst})$ \\
\hline
\end{tabular}
counting here the $\mathrm{D}^{*+}$, which feeds into the $\mathrm{D}^{0}$ and $\mathrm{D}^{+}$mesons.
In the left panel of Fig. 2 the fractions $f\left(\mathrm{c} \rightarrow \mathrm{H}_{\mathrm{c}}\right)$ are compared with values derived from experimental measurements performed in $\mathrm{e}^{+} \mathrm{e}^{-}$collisions at LEP and $\mathrm{B}$ factories as well as in ep collisions [63]. The fragmentation fractions measured at midrapidity in pp collisions at the LHC are different from the ones measured in $\mathrm{e}^{+} \mathrm{e}^{-}$and ep collisions, confirming significant evidence that the assumption of universality (collision-system independence) of partonto-hadron fragmentation is not valid as reported in $[4,24,28]$. The fractions $f\left(\mathrm{c} \rightarrow \mathrm{H}_{\mathrm{c}}\right)$ measured in $\mathrm{e}^{+} \mathrm{e}^{-}$, including the $\Lambda_{\mathrm{c}}^{+}$baryon, are in agreement with a standard canonical SHM [64]. The $\Lambda_{\mathrm{c}}^{+} / \mathrm{D}^{0}$ ratio measured at midrapidity in $\mathrm{pp}$ and $\mathrm{p}-\mathrm{Pb}$ collisions at the LHC is different from the one measured at forward rapidity by the $\mathrm{LHCb}$ Collaboration $[8,65]$ as discussed in $[23,25]$.

An increase of about a factor 3.3 for the fragmentation fractions for the $\Lambda_{\mathrm{c}}^{+}$baryons with respect to $\mathrm{e}^{+} \mathrm{e}^{-}$and ep collisions, and a concomitant decrease of about a factor 1.4-1.2 for the D mesons are observed. The significance of the difference considering the uncertainties of both measurements is about $5 \sigma$ for $\Lambda_{c}^{+}$baryons. This in turn decreases the fragmentation into $\mathrm{D}^{0}$ mesons at midrapidity by $6 \sigma$ with respect to the measurements in $\mathrm{e}^{+} \mathrm{e}^{-}$and ep collisions. In previous measurements in $\mathrm{e}^{+} \mathrm{e}^{-}$and ep collisions, no value for the $\Xi_{\mathrm{c}}^{0}$ was obtained, and the yield was estimated according to the assumption $f\left(\mathrm{c} \rightarrow \Xi_{\mathrm{c}}^{+}\right) /$ $f\left(\mathrm{c} \rightarrow \Lambda_{\mathrm{c}}^{+}\right)=f\left(\mathrm{~s} \rightarrow \Xi^{-}\right) / f\left(\mathrm{~s} \rightarrow \Lambda^{0}\right) \sim 0.004$ [63]. The fraction $f\left(\mathrm{c} \rightarrow \Xi_{\mathrm{c}}^{0}\right)$ was measured for the first time, and $f\left(\mathrm{c} \rightarrow \Xi_{\mathrm{c}}^{0}\right) / f\left(\mathrm{c} \rightarrow \Lambda_{\mathrm{c}}^{+}\right)=0.39 \pm 0.07(\mathrm{stat})_{-0.07}^{+0.08}($ syst $)$ was found [28]. A first attempt to compute the fragmentation fractions in pp collisions at the LHC was performed in [63] assuming universal fragmentation, since at that time the measurements of charm baryons at midrapidity were not yet available. The measurements reported here challenge that assumption.

The updated fragmentation fractions, obtained for the first time taking into account the measurements of $\mathrm{D}^{0}, \mathrm{D}^{+}$, $\mathrm{D}_{\mathrm{s}}^{+}, \Lambda_{\mathrm{c}}^{+}$, and $\Xi_{\mathrm{c}}^{0}$ at midrapidity in pp collisions at $\sqrt{s}=5.02 \mathrm{TeV}$, allowed the recomputation of the charm production cross sections per unit of rapidity at midrapidity in pp collisions at $\sqrt{s}=2.76$ and $7 \mathrm{TeV}$. The $\Lambda_{\mathrm{c}}^{+} / \mathrm{D}^{0}$ ratios measured in pp at different collision energies, as well as the $\Xi_{\mathrm{c}}^{0} / \mathrm{D}^{0}$ ratio, are compatible $[25,28,56]$. The charm cross sections were obtained by scaling the $p_{\mathrm{T}}$-integrated $\mathrm{D}^{0}$ meson cross section [1,3] for the relative fragmentation fraction of a charm quark into a $\mathrm{D}^{0}$ meson measured in $\mathrm{pp}$ collisions at $\sqrt{s}=5.02 \mathrm{TeV}$ and applying the two correction factors for the different shapes of the rapidity distributions of charm hadrons and $c \bar{c}$ pairs. The $p_{\mathrm{T}}$-integrated $\mathrm{D}^{0}$-meson cross section was used because at the other energies not all charm hadrons were measured and the $\mathrm{D}^{0}$ measurements are the most precise. The uncertainties of the FF were taken into account in calculating the cc production cross section, as was the uncertainty introduced by the 

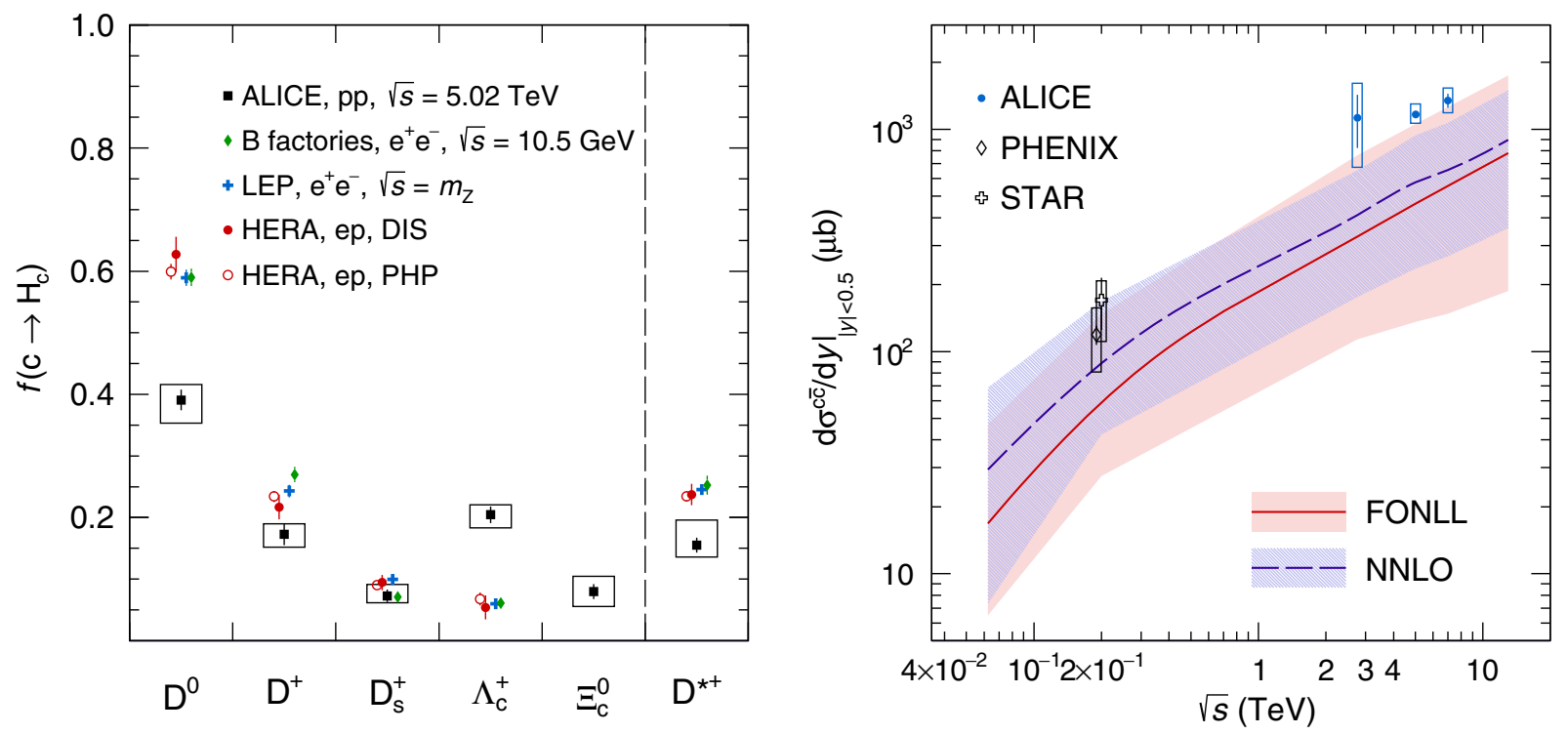

FIG. 2. Left: charm-quark fragmentation fractions into charm hadrons measured in pp collisions at $\sqrt{s}=5.02$ TeV in comparison with experimental measurements performed in $\mathrm{e}^{+} \mathrm{e}^{-}$collisions at LEP and at B factories, and in ep collisions at HERA [63]. The $\mathrm{D}^{*+}$ meson is depicted separately since its contribution is also included in the ground-state charm mesons. Right: charm production cross section at midrapidity per unit of rapidity as a function of the collision energy. STAR [11] and PHENIX [66] results, slightly displaced in the horizontal direction for better visibility, are reported. Comparisons with FONLL [13-15] (red band) and NNLO [67-69] (violet band) pQCD calculations are also shown.

rapidity correction factors. The $\mathrm{BR}$ of the $\mathrm{D}^{0} \rightarrow \mathrm{K}^{-} \pi^{+}$ decay channel was also updated, considering the latest value reported in the PDG [47].

The resulting $c \bar{c}$ cross sections per unit of rapidity at midrapidity are

$$
\begin{aligned}
\left.\frac{\mathrm{d} \sigma^{\mathrm{c} \overline{\mathrm{c}}}}{\mathrm{d} y}\right|_{|y|<0.5} ^{\mathrm{pp}, 7 \mathrm{TeV}}= & 1347 \pm 97(\text { stat }) \pm 104(\text { syst }) \pm 11(\mathrm{BR}) \\
& \pm_{-105}^{+142}(\mathrm{FF}) \pm 44(\mathrm{RS}) \pm 47(\text { lumi }) \mu \mathrm{b}
\end{aligned}
$$

and

$$
\begin{aligned}
\left.\frac{\mathrm{d} \sigma^{\mathrm{c} \overline{\mathrm{c}}}}{\mathrm{d} y}\right|_{|y|<0.5} ^{\mathrm{pp}, 2.76 \mathrm{TeV}} & =1126 \pm 303(\mathrm{stat})_{-429}^{+258}(\mathrm{syst})_{-53}^{+397}(\mathrm{extr}) \\
& \pm 9(\mathrm{BR}) \pm_{-88}^{+119}(\mathrm{FF}) \pm 61(\mathrm{RS}) \pm 21(\text { lumi }) \mu \mathrm{b}
\end{aligned}
$$

for $\sqrt{s}=7$ and $2.76 \mathrm{TeV}$, respectively. The updated $\mathrm{c} \overline{\mathrm{c}}$ cross sections at $\sqrt{s}=2.76$ and $7 \mathrm{TeV}$ are about $40 \%$ higher than the previously published results [1,3], reflecting the differences in the fragmentation into charm baryons measured in $\mathrm{e}^{+} \mathrm{e}^{-}$and pp collisions.

In the right panel of Fig. 2, the measured cc cross sections are compared with FONLL and NNLO predictions as a function of the collision energy. The NNLO values were obtained by the authors of $[67,68]$ by applying to the central value of the FONLL $\mathrm{d} \sigma^{\mathrm{cc}} / \mathrm{d} y$ a $K$ factor (NNLO/ NLO) calculated with a modified version of the top ++ code [69] with parameter values as in $[67,68]$ and using the relative scale uncertainties obtained at NNLO with top ++ . The cic cross sections are also compared with the STAR [11] and PHENIX [66] results measured in pp collisions at $\sqrt{s}=200 \mathrm{GeV}$. The STAR measurement is obtained by scaling the $\mathrm{D}^{0}$ and $\mathrm{D}^{*+}$ cross sections by the charm-quark fragmentation fractions measured in $\mathrm{e}^{+} \mathrm{e}^{-}$ collisions from the CLEO and BELLE experiments [63]. The PHENIX $c \bar{c}$ cross section is obtained from the measurement of the cross sections of electrons from semileptonic heavy-flavor hadron decays. Both results are compatible within uncertainties with the upper edge of the FONLL and NNLO bands. The cī cross sections measured at the three LHC collision energies are higher than the upper edge of the FONLL and NNLO bands; however, they are compatible within approximately 1 standard deviation of the experimental uncertainty. The theoretical uncertainties are estimated as a convolution of the $\mathrm{pQCD}$ calculations obtained by varying the factorization and renormalization scales. The uncertainties of the PDFs and of the charm-quark mass are also included in the uncertainties of both calculations and are determined with FONLL as described in [15].

In summary, the charm production cross section per unit of rapidity at midrapidity in $\mathrm{pp}$ collisions at $\sqrt{s}=$ $5.02 \mathrm{TeV}$ was determined by exploiting recent measurements of the ground-state charm hadrons, including, for the first time, the measured baryon states. The charm fragmentation fractions $f\left(\mathrm{c} \rightarrow \mathrm{H}_{\mathrm{c}}\right)$ were computed for the first time in hadron collisions at the LHC using measurements 
of charm baryons at midrapidity, and they were found to be different from those measured in $\mathrm{e}^{+} \mathrm{e}^{-}$and ep collisions. This observation indicates that the hadronization of charm quarks into charm hadrons is not a universal process among different collision systems. The fragmentation fraction for the $\Xi_{\mathrm{c}}^{0}$ baryon was measured for the first time and found to be sizable. Finally, the charm production cross section per unit of rapidity at midrapidity, in pp collisions at $\sqrt{s}=5.02 \mathrm{TeV}$ at the LHC, was measured and lies at the upper edge of the theoretical pQCD calculations.

\section{ACKNOWLEDGEMENTS}

The ALICE Collaboration would like to thank all its engineers and technicians for their invaluable contributions to the construction of the experiment and the CERN accelerator teams for the outstanding performance of the LHC complex. The ALICE Collaboration gratefully acknowledges the resources and support provided by all Grid centers and the Worldwide LHC Computing Grid (WLCG) Collaboration. The ALICE Collaboration acknowledges the following funding agencies for their support in building and running the ALICE detector: A. I. Alikhanyan National Science Laboratory (Yerevan Physics Institute) Foundation (ANSL), State Committee of Science and World Federation of Scientists (WFS), Armenia; Austrian Academy of Sciences, Austrian Science Fund (FWF): [M 2467-N36] and Nationalstiftung für Forschung, Technologie und Entwicklung, Austria; Ministry of Communications and High Technologies, National Nuclear Research Center, Azerbaijan; Conselho Nacional de Desenvolvimento Científico e Tecnológico (CNPq), Financiadora de Estudos e Projetos (Finep), Fundação de Amparo à Pesquisa do Estado de São Paulo (FAPESP) and Universidade Federal do Rio Grande do Sul (UFRGS), Brazil; Ministry of Education of China (MOEC), Ministry of Science \& Technology of China (MSTC) and National Natural Science Foundation of China (NSFC), China; Ministry of Science and Education and Croatian Science Foundation, Croatia; Centro de Aplicaciones Tecnológicas y Desarrollo Nuclear (CEADEN), Cubaenergía, Cuba; Ministry of Education, Youth and Sports of the Czech Republic, Czech Republic; The Danish Council for Independent Research I Natural Sciences, the VILLUM FONDEN and Danish National Research Foundation (DNRF), Denmark; Helsinki Institute of Physics (HIP), Finland; Commissariat à l'Energie Atomique (CEA) and Institut National de Physique Nucléaire et de Physique des Particules (IN2P3) and Centre National de la Recherche Scientifique (CNRS), France; Bundesministerium für Bildung und Forschung (BMBF) and GSI Helmholtz zentrum für Schwerionenforschung $\mathrm{GmbH}$, Germany;
General Secretariat for Research and Technology, Ministry of Education, Research and Religions, Greece; National Research, Development and Innovation Office, Hungary; Department of Atomic Energy Government of India (DAE), Department of Science and Technology, Government of India (DST), University Grants Commission, Government of India (UGC) and Council of Scientific and Industrial Research (CSIR), India; Indonesian Institute of Science, Indonesia; Istituto Nazionale di Fisica Nucleare (INFN), Italy; Institute for Innovative Science and Technology, Nagasaki Institute of Applied Science (IIST), Japanese Ministry of Education, Culture, Sports, Science and Technology (MEXT) and Japan Society for the Promotion of Science (JSPS) KAKENHI, Japan; Consejo Nacional de Ciencia (CONACYT) y Tecnología, through Fondo de Cooperación Internacional en Ciencia y Tecnología (FONCICYT) and Dirección General de Asuntos del Personal Academico (DGAPA), Mexico; Nederlandse Organisatie voor Wetenschappelijk Onderzoek (NWO), Netherlands; The Research Council of Norway, Norway; Commission on Science and Technology for Sustainable Development in the South (COMSATS), Pakistan; Pontificia Universidad Católica del Perú, Peru; Ministry of Education and Science, National Science Centre and WUT ID-UB, Poland; Korea Institute of Science and Technology Information and National Research Foundation of Korea (NRF), Republic of Korea; Ministry of Education and Scientific Research, Institute of Atomic Physics and Ministry of Research and Innovation and Institute of Atomic Physics, Romania; Joint Institute for Nuclear Research (JINR), Ministry of Education and Science of the Russian Federation, National Research Centre Kurchatov Institute, Russian Science Foundation and Russian Foundation for Basic Research, Russia; Ministry of Education, Science, Research and Sport of the Slovak Republic, Slovakia; National Research Foundation of South Africa, South Africa; Swedish Research Council (VR) and Knut \& Alice Wallenberg Foundation (KAW), Sweden; European Organization for Nuclear Research, Switzerland; Suranaree University of Technology (SUT), National Science and Technology Development Agency (NSDTA) and Office of the Higher Education Commission under NRU project of Thailand, Thailand; Turkish Energy, Nuclear and Mineral Research Agency (TENMAK), Turkey; National Academy of Sciences of Ukraine, Ukraine; Science and Technology Facilities Council (STFC), United Kingdom; the U.S. National Science Foundation (NSF) and the U.S. Department of Energy, Office of Nuclear Physics (DOE NP), USA. 
[1] B. Abelev et al. (ALICE Collaboration), Measurement of charm production at central rapidity in proton-proton collisions at $\sqrt{s}=2.76 \mathrm{TeV}$, J. High Energy Phys. 07 (2012) 191.

[2] B. Abelev et al. (ALICE Collaboration), $\mathrm{D}_{\mathrm{s}}^{+}$meson production at central rapidity in proton-proton collisions at $\sqrt{s}=7 \mathrm{TeV}$, Phys. Lett. B 718, 279 (2012).

[3] S. Acharya et al. (ALICE Collaboration), Measurement of D-meson production at mid-rapidity in $\mathrm{pp}$ collisions at $\sqrt{s}=7$ TeV, Eur. Phys. J. C 77, 550 (2017).

[4] S. Acharya et al. (ALICE Collaboration), Measurement of $\mathrm{D}^{0}, \mathrm{D}^{+}, \mathrm{D}^{*+}$ and $\mathrm{D}_{\mathrm{s}}^{+}$production in $\mathrm{pp}$ collisions at $\sqrt{s}=5.02 \mathrm{TeV}$ with ALICE, Eur. Phys. J. C 79, 388 (2019).

[5] S. Acharya et al. (ALICE Collaboration), Measurement of beauty and charm production in pp collisions at $\sqrt{s}=5.02 \mathrm{TeV}$ via non-prompt and prompt $\mathrm{D}$ mesons, J. High Energy Phys. 05 (2021) 220.

[6] G. Aad et al. (ATLAS Collaboration), Measurement of $\mathrm{D}^{* \pm}, \mathrm{D}^{ \pm}$and $\mathrm{D}_{\mathrm{s}}^{ \pm}$meson production cross sections in $p p$ collisions at $\sqrt{s}=7 \mathrm{TeV}$ with the ATLAS detector, Nucl. Phys. B907, 717 (2016).

[7] A. M. Sirunyan et al. (CMS Collaboration), Nuclear modification factor of $\mathrm{D}^{0}$ mesons in $\mathrm{PbPb}$ collisions at $\sqrt{s_{\mathrm{NN}}}=5.02 \mathrm{TeV}$, Phys. Lett. B 782, 474 (2018).

[8] R. Aaij et al. (LHCb Collaboration), Prompt charm production in pp collisions at $\sqrt{(} s)=7 \mathrm{TeV}$, Nucl. Phys. B871, 1 (2013).

[9] R. Aaij et al. (LHCb Collaboration), Measurements of prompt charm production cross-sections in $p p$ collisions at $\sqrt{s}=13 \mathrm{TeV}$, J. High Energy Phys. 03 (2016) 159; 09 (2016) 013(E); 05 (2017) 074(E).

[10] R. Aaij et al. (LHCb Collaboration), Measurements of prompt charm production cross-sections in pp collisions at $\sqrt{s}=5 \mathrm{TeV}$, J. High Energy Phys. 06 (2017) 147.

[11] L. Adamczyk et al. (STAR Collaboration), Measurements of $D^{0}$ and $D^{*}$ production in $p+p$ collisions at $\sqrt{s}=200 \mathrm{GeV}$, Phys. Rev. D 86, 072013 (2012).

[12] D. Acosta et al. (CDF Collaboration), Measurement of Prompt Charm Meson Production Cross Sections in $p \bar{p}$ Collisions at $\sqrt{s}=1.96 \mathrm{TeV}$, Phys. Rev. Lett. 91, 241804 (2003).

[13] M. Cacciari, M. Greco, and P. Nason, The $p_{T}$ spectrum in heavy-flavour hadroproduction, J. High Energy Phys. 05 (1998) 007.

[14] M. Cacciari, S. Frixione, and P. Nason, The $p_{T}$ spectrum in heavy-flavour photoproduction, J. High Energy Phys. 03 (2001) 006.

[15] M. Cacciari, S. Frixione, N. Houdeau, M. L. Mangano, P. Nason, and G. Ridolfi, Theoretical predictions for charm and bottom production at the LHC, J. High Energy Phys. 10 (2012) 137.

[16] B. A. Kniehl, G. Kramer, I. Schienbein, and H. Spiesberger, Inclusive $D^{* \pm}$ production in $p \bar{p}$ collisions with massive charm quarks, Phys. Rev. D 71, 014018 (2005).

[17] B. Kniehl, G. Kramer, I. Schienbein, and H. Spiesberger, Inclusive charmed-meson production at the CERN LHC, Eur. Phys. J. C 72, 2082 (2012).

[18] M. Benzke, M. Garzelli, B. Kniehl, G. Kramer, S. Moch, and G. Sigl, Prompt neutrinos from atmospheric charm in the general-mass variable-flavor-number scheme, J. High Energy Phys. 12 (2017) 021.

[19] G. Kramer and H. Spiesberger, Study of heavy meson production in $\mathrm{p}-\mathrm{Pb}$ collisions at $\sqrt{s_{\mathrm{NN}}}=5.02 \mathrm{TeV}$ in the general-mass variable-flavour-number scheme, Nucl. Phys. B925, 415 (2017).

[20] I. Helenius and H. Paukkunen, Revisiting the D-meson hadroproduction in general-mass variable flavour number scheme, J. High Energy Phys. 05 (2018) 196.

[21] E. Braaten, K.-M. Cheung, S. Fleming, and T. C. Yuan, Perturbative QCD fragmentation functions as a model for heavy quark fragmentation, Phys. Rev. D 51, 4819 (1995).

[22] L. Gladilin, Fragmentation fractions of $c$ and $b$ quarks into charmed hadrons at LEP, Eur. Phys. J. C 75, 19 (2015).

[23] S. Acharya et al. (ALICE Collaboration), $\Lambda_{\mathrm{c}}^{+}$production in $\mathrm{pp}$ collisions at $\sqrt{s}=7 \mathrm{TeV}$ and in $\mathrm{p}-\mathrm{Pb}$ collisions at $\sqrt{s_{\mathrm{NN}}}=5.02 \mathrm{TeV}$, J. High Energy Phys. 04 (2018) 108.

[24] S. Acharya et al. (ALICE Collaboration), $\Lambda_{\mathrm{c}}^{+}$Production and Baryon-to-Meson Ratios in $\mathrm{pp}$ and $\mathrm{p}-\mathrm{Pb}$ Collisions at $\sqrt{s_{\mathrm{NN}}}=5.02 \mathrm{TeV}$ at the LHC, Phys. Rev. Lett. 127, 202301 (2021).

[25] S. Acharya et al. (ALICE Collaboration), $\Lambda_{\mathrm{c}}^{+}$production in pp and in $\mathrm{p}-\mathrm{Pb}$ collisions at $\sqrt{s_{\mathrm{NN}}}=5.02 \mathrm{TeV}$, Phys. Rev. C 104, 054905 (2021).

[26] A. M. Sirunyan et al. (CMS Collaboration), Production of $\Lambda_{\mathrm{c}}^{+}$baryons in proton-proton and lead-lead collisions at $\sqrt{s_{\mathrm{NN}}}=5.02 \mathrm{TeV}$, Phys. Lett. B 803, 135328 (2020).

[27] S. Acharya et al. (ALICE Collaboration), First measurement of $\Xi_{\mathrm{c}}^{0}$ production in pp collisions at $\sqrt{\mathbf{s}}=7 \mathrm{TeV}$, Phys. Lett. B 781, 8 (2018).

[28] S. Acharya et al. (ALICE Collaboration), Measurement of the production cross section of prompt $\Xi_{\mathrm{c}}^{0}$ baryons at midrapidity in pp collisions at $\sqrt{s}=5.02 \mathrm{TeV}$, J. High Energy Phys. 10 (2021) 159.

[29] S. Acharya et al. (ALICE Collaboration), Measurement of the cross sections of $\Xi_{\mathrm{c}}^{0}$ and $\Xi_{\mathrm{c}}^{+}$baryons and branchingfraction ratio $\operatorname{BR}\left(\Xi_{\mathrm{c}}^{0} \rightarrow \Xi^{-} \mathrm{e}^{+} \nu_{\mathrm{e}}\right) / \mathrm{BR}\left(\Xi_{\mathrm{c}}^{0} \rightarrow \Xi^{-} \pi^{+}\right)$in pp collisions at $13 \mathrm{TeV}$, Phys. Rev. Lett. 127, 272001 (2021).

[30] T. Aaltonen et al. (CDF Collaboration), Measurement of ratios of fragmentation fractions for bottom hadrons in $p \bar{p}$ collisions at $\sqrt{s}=1.96-\mathrm{TeV}$, Phys. Rev. D 77, 072003 (2008).

[31] R. Aaij et al. (LHCb Collaboration), Measurement of $b$-hadron production fractions in $7 \mathrm{TeV}$ pp collisions, Phys. Rev. D 85, 032008 (2012).

[32] R. Aaij et al. (LHCb Collaboration), Measurement of $b$ hadron fractions in $13 \mathrm{TeV} p p$ collisions, Phys. Rev. D 100, 031102 (2019).

[33] J. Song, H.-h. Li, and F.-l. Shao, New feature of low $p_{T}$ charm quark hadronization in $p p$ collisions at $\sqrt{s}=7 \mathrm{TeV}$, Eur. Phys. J. C 78, 344 (2018).

[34] V. Minissale, S. Plumari, and V. Greco, Charm hadrons in pp collisions at LHC energy within a coalescence plus fragmentation approach, Phys. Lett. B 821, 136622 (2021).

[35] M. He and R. Rapp, Charm-baryon production in protonproton collisions, Phys. Lett. B 795, 117 (2019).

[36] J. R. Christiansen and P. Z. Skands, String formation beyond leading colour, J. High Energy Phys. 08 (2015) 003.

[37] R. Maciuła and A. Szczurek, Production of $\Lambda_{c}$ baryons at the LHC within the $k_{T}$-factorization approach and independent 
parton fragmentation picture, Phys. Rev. D 98, 014016 (2018).

[38] B. A. Kniehl, G. Kramer, I. Schienbein, and H. Spiesberger, $\Lambda_{c}^{ \pm}$production in pp collisions with a new fragmentation function, Phys. Rev. D 101, 114021 (2020).

[39] P. Braun-Munzinger and J. Stachel, (Non)thermal aspects of charmonium production and a new look at $\mathrm{J} / \psi$ suppression, Phys. Lett. B 490, 196 (2000).

[40] X. Zhao and R. Rapp, Medium modifications and production of charmonia at LHC, Nucl. Phys. A859, 114 (2011).

[41] Y.-P. Liu, Z. Qu, N. Xu, and P.-F. Zhuang, J/ $\psi$ transverse momentum distribution in high energy nuclear collisions at RHIC, Phys. Lett. B 678, 72 (2009).

[42] J. Adam et al. (ALICE Collaboration), $\mathrm{J} / \psi$ suppression at forward rapidity in $\mathrm{Pb}-\mathrm{Pb}$ collisions at $\sqrt{\mathbf{s}_{\mathrm{NN}}}=\mathbf{5 . 0 2} \mathrm{TeV}$, Phys. Lett. B 766, 212 (2017).

[43] S. Acharya et al. (ALICE Collaboration), Centrality and transverse momentum dependence of inclusive $\mathrm{J} / \psi$ production at midrapidity in $\mathrm{Pb}-\mathrm{Pb}$ collisions at $\sqrt{s_{\mathrm{NN}}}=5.02 \mathrm{TeV}$, Phys. Lett. B 805, 135434 (2020).

[44] K. Aamodt et al. (ALICE Collaboration), The ALICE experiment at the CERN LHC, J. Instrum. 3, S08002 (2008).

[45] B. B. Abelev et al. (ALICE Collaboration), Performance of the ALICE experiment at the CERN LHC, Int. J. Mod. Phys. A 29, 1430044 (2014).

[46] ALICE Collaboration, ALICE 2017 luminosity determination for pp collisions at $\sqrt{s}=5 \mathrm{TeV}$, http://cds.cern.ch/ record/2648933.

[47] P. Zyla et al. (Particle Data Group), Review of particle physics, Prog. Theor. Exp. Phys. 2020, 083 C01 (2020).

[48] S. Acharya et al. (ALICE Collaboration), Inclusive $\mathrm{J} / \psi$ production at mid-rapidity in $\mathrm{pp}$ collisions at $\sqrt{s}=5.02 \mathrm{TeV}$, J. High Energy Phys. 10 (2019) 084.

[49] P. Skands, S. Carrazza, and J. Rojo, Tuning PYTHIA 8.1: The Monash 2013 tune, Eur. Phys. J. C 74, 3024 (2014).

[50] S. Chatrchyan et al. (CMS Collaboration), $J / \psi$ and $\psi(2 S)$ production in pp collisions at $\sqrt{s}=7 \mathrm{TeV}$, J. High Energy Phys. 02 (2012) 011.

[51] G. Aad et al. (ATLAS Collaboration), Measurement of the differential cross-sections of inclusive, prompt and nonprompt $J / \psi$ production in proton-proton collisions at $\sqrt{s}=7 \mathrm{TeV}$, Nucl. Phys. B850, 387 (2011).

[52] B. Abelev et al. (ALICE Collaboration), Measurement of prompt $J / \psi$ and beauty hadron production cross sections at mid-rapidity in $p p$ collisions at $\sqrt{s}=7 \mathrm{TeV}$, J. High Energy Phys. 11 (2012) 065.

[53] D. Ebert, R. Faustov, and V. Galkin, Spectroscopy and Regge trajectories of heavy baryons in the relativistic quarkdiquark picture, Phys. Rev. D 84, 014025 (2011).

[54] A. Andronic, P. Braun-Munzinger, K. Redlich, and J. Stachel, Decoding the phase structure of QCD via particle production at high energy, Nature (London) 561, 321 (2018).

[55] J. Cleymans, P. M. Lo, K. Redlich, and N. Sharma, Multiplicity dependence of (multi)strange baryons in the canonical ensemble with phase shift corrections, Phys. Rev. C 103, 014904 (2021).

[56] S. Acharya et al. (ALICE Collaboration), Measurement of prompt $\mathrm{D}^{0}, \Lambda_{c}^{+}$, and $\Sigma_{c}^{0,++}$ (2455) production in $\mathrm{pp}$ collisions at $\sqrt{s}=13 \mathrm{TeV}$, Phys. Rev. Lett. 128, 012001 (2022).

[57] S. Frixione, P. Nason, and G. Ridolfi, A Positive-weight next-to-leading-order Monte Carlo for heavy flavour hadroproduction, J. High Energy Phys. 09 (2007) 126.

[58] H.-L. Lai, M. Guzzi, J. Huston, Z. Li, P. M. Nadolsky, J. Pumplin, and C.-P. Yuan, New parton distributions for collider physics, Phys. Rev. D 82, 074024 (2010).

[59] S. Dulat, T.-J. Hou, J. Gao, M. Guzzi, J. Huston, P. Nadolsky, J. Pumplin, C. Schmidt, D. Stump, and C. Yuan, New parton distribution functions from a global analysis of quantum chromodynamics, Phys. Rev. D 93, 033006 (2016).

[60] T.-J. Hou et al., New CTEQ global analysis of quantum chromodynamics with high-precision data from the LHC, Phys. Rev. D 103, 014013 (2021).

[61] P. M. Nadolsky, H.-L. Lai, Q.-H. Cao, J. Huston, J. Pumplin, D. Stump, W.-K. Tung, and C. P. Yuan, Implications of CTEQ global analysis for collider observables, Phys. Rev. D 78, 013004 (2008).

[62] S. Forte and S. Carrazza, Parton distribution functions, arXiv:2008.12305.

[63] M. Lisovyi, A. Verbytskyi, and O. Zenaiev, Combined analysis of charm-quark fragmentation-fraction measurements, Eur. Phys. J. C 76, 397 (2016).

[64] A. Andronic, F. Beutler, P. Braun-Munzinger, K. Redlich, and J. Stachel, Statistical hadronization of heavy flavor quarks in elementary collisions: Successes and failures, Phys. Lett. B 678, 350 (2009).

[65] R. Aaij et al. (LHCb Collaboration), Prompt $\Lambda_{c}^{+}$production in $p \mathrm{~Pb}$ collisions at $\sqrt{s_{N N}}=5.02 \mathrm{TeV}$, J. High Energy Phys. 02 (2019) 102.

[66] A. Adare et al. (PHENIX Collaboration), Heavy quark production in $p+p$ and energy loss and flow of heavy quarks in $\mathrm{Au}+\mathrm{Au}$ collisions at $\sqrt{s_{N N}}=200 \mathrm{GeV}$, Phys. Rev. C 84, 044905 (2011).

[67] D. d'Enterria and A. M. Snigirev, Triple Parton Scatterings in High-Energy Proton-Proton Collisions, Phys. Rev. Lett. 118, 122001 (2017).

[68] D. d'Enterria and A. M. Snigirev, Triple-parton scatterings in proton-nucleus collisions at high energies, Eur. Phys. J. C 78, 359 (2018).

[69] M. Czakon, P. Fiedler, and A. Mitov, Total Top-Quark PairProduction Cross Section at Hadron Colliders through $O\left(\alpha_{S}^{4}\right)$, Phys. Rev. Lett. 110, 252004 (2013).

\footnotetext{
S. Acharya, ${ }^{143}$ D. Adamová, ${ }^{98}$ A. Adler, ${ }^{76}$ J. Adolfsson, ${ }^{83}$ G. Aglieri Rinella, ${ }^{35}$ M. Agnello, ${ }^{31}$ N. Agrawal,${ }^{55}$ Z. Ahammed, ${ }^{143}$ S. Ahmad, ${ }^{16}$ S. U. Ahn, ${ }^{78}$ I. Ahuja, ${ }^{39}$ Z. Akbar, ${ }^{52}$ A. Akindinov, ${ }^{95}$ M. Al-Turany, ${ }^{110}$ S. N. Alam, ${ }^{41}$
} 
D. Aleksandrov, ${ }^{91}$ B. Alessandro, ${ }^{61}$ H. M. Alfanda, ${ }^{7}$ R. Alfaro Molina, ${ }^{73}$ B. Ali, ${ }^{16}$ Y. Ali, ${ }^{14}$ A. Alici, ${ }^{26}$ N. Alizadehvandchali, ${ }^{127}$ A. Alkin, ${ }^{35}$ J. Alme, ${ }^{21}$ T. Alt,${ }^{70}$ L. Altenkamper, ${ }^{21}$ I. Altsybeev, ${ }^{115}$ M. N. Anaam, ${ }^{7}$ C. Andrei, ${ }^{49}$ D. Andreou, ${ }^{93}$ A. Andronic, ${ }^{146} \mathrm{M}$. Angeletti, ${ }^{35} \mathrm{~V}$. Anguelov, ${ }^{107} \mathrm{~F}$. Antinori, ${ }^{58} \mathrm{P}$. Antonioli, ${ }^{55} \mathrm{C}$. Anuj, ${ }^{16} \mathrm{~N}$. Apadula, ${ }^{82}$ L. Aphecetche, ${ }^{117}$ H. Appelshäuser, ${ }^{70}$ S. Arcelli, ${ }^{26}$ R. Arnaldi, ${ }^{61}$ I. C. Arsene,${ }^{20}$ M. Arslandok,${ }^{107,148}$ A. Augustinus, ${ }^{35}$ R. Averbeck, ${ }^{110}$ S. Aziz, ${ }^{80}$ M. D. Azmi, ${ }^{16}$ A. Badală,${ }^{57}$ Y. W. Baek, ${ }^{42}$ X. Bai, ${ }^{110,131}$ R. Bailhache,${ }^{70}$ Y. Bailung, ${ }^{51}$ R. Bala, ${ }^{104}$ A. Balbino, ${ }^{31}$ A. Baldisseri, ${ }^{140}$ B. Balis,${ }^{2}$ M. Ball, ${ }^{44}$ D. Banerjee, ${ }^{4}$ R. Barbera, ${ }^{27}$ L. Barioglio,${ }^{25,108}$ M. Barlou, ${ }^{87}$ G. G. Barnaföldi, ${ }^{147}$ L. S. Barnby, ${ }^{97}$ V. Barret, ${ }^{137}$ C. Bartels, ${ }^{130}$ K. Barth, ${ }^{35}$ E. Bartsch,${ }^{70}$ F. Baruffaldi,${ }^{28}$ N. Bastid, ${ }^{137}$ S. Basu, ${ }^{83}$ G. Batigne, ${ }^{117}$ B. Batyunya ${ }^{77}$ D. Bauri, ${ }^{50}$ J. L. Bazo Alba, ${ }^{114}$ I. G. Bearden, ${ }^{92}$ C. Beattie,${ }^{148}$ I. Belikov, ${ }^{139}$ A. D. C. Bell Hechavarria, ${ }^{146}$ F. Bellini, ${ }^{26,35}$ R. Bellwied, ${ }^{127}$ S. Belokurova, ${ }^{115}$ V. Belyaev, ${ }^{96}$ G. Bencedi, ${ }^{71}$ S. Beole, ${ }^{25}$ A. Bercuci, ${ }^{49}$ Y. Berdnikov, ${ }^{101}$ A. Berdnikova, ${ }^{107}$ D. Berenyi, ${ }^{147}$ L. Bergmann, ${ }^{107}$ M. G. Besoiu, ${ }^{69}$ L. Betev,${ }^{35}$ P. P. Bhaduri, ${ }^{143}$ A. Bhasin, ${ }^{104}$ M. A. Bhat, ${ }^{4}$ B. Bhattacharjee, ${ }^{43}$ P. Bhattacharya,${ }^{23}$ L. Bianchi, ${ }^{25}$ N. Bianchi, ${ }^{53}$ J. Bielčík, ${ }^{38}$ J. Bielč́́ková, ${ }^{98}$ J. Biernat, ${ }^{120}$ A. Bilandzic, ${ }^{108}$ G. Biro, ${ }^{147}$ S. Biswas, J. T. Blair, ${ }^{121}$ D. Blau, ${ }^{91}$ M. B. Blidaru, ${ }^{110}$ C. Blume ${ }^{70}$ G. Boca, ${ }^{29,59}$ F. Bock, ${ }^{99}$ A. Bogdanov, ${ }^{96}$ S. Boi, ${ }^{23}$ J. Bok, ${ }^{63}$ L. Boldizsár, ${ }^{147}$ A. Bolozdynya, ${ }^{96}$ M. Bombara,,${ }^{39}$ P. M. Bond ${ }^{35}$ G. Bonomi, ${ }^{59,142} \mathrm{H}$. Borel,${ }^{140}$ A. Borissov,${ }^{84} \mathrm{H}$. Bossi, ${ }^{148}$ E. Botta, ${ }^{25}$ L. Bratrud ${ }^{70}$ P. Braun-Munzinger, ${ }^{110}$ M. Bregant, ${ }^{123}$ M. Broz,${ }^{38}$ G. E. Bruno, ${ }^{34,109}$ M. D. Buckland, ${ }^{130}$ D. Budnikov, ${ }^{111}$ H. Buesching, ${ }^{70}$ S. Bufalino, ${ }^{31}$ O. Bugnon, ${ }^{117}$ P. Buhler, ${ }^{116}$ Z. Buthelezi, ${ }^{74,134}$ J. B. Butt, ${ }^{14}$ S. A. Bysiak, ${ }^{120}$ D. Caffarri, ${ }^{93}$ M. Cai, ${ }^{7,28}$ H. Caines, ${ }^{148}$ A. Caliva, ${ }^{110}$ E. Calvo Villar, ${ }^{114}$ J. M. M. Camacho, ${ }^{122}$ R. S. Camacho, ${ }^{46}$ P. Camerini, ${ }^{24}$ F. D. M. Canedo, ${ }^{123}$ F. Carnesecchi, ${ }^{26,35}$ R. Caron, ${ }^{140}$

J. Castillo Castellanos, ${ }^{140}$ E. A. R. Casula, ${ }^{23}$ F. Catalano, ${ }^{31}$ C. Ceballos Sanchez,${ }^{77}$ P. Chakraborty, ${ }^{50}$ S. Chandra, ${ }^{143}$ S. Chapeland, ${ }^{35}$ M. Chartier, ${ }^{130}$ S. Chattopadhyay, ${ }^{143}$ S. Chattopadhyay, ${ }^{112}$ A. Chauvin, ${ }^{23}$ T. G. Chavez, ${ }^{46}$ C. Cheshkov, ${ }^{138}$ B. Cheynis, ${ }^{138}$ V. Chibante Barroso, ${ }^{35}$ D. D. Chinellato, ${ }^{124}$ S. Cho, ${ }^{63}$ P. Chochula, ${ }^{35}$ P. Christakoglou, ${ }^{93}$ C. H. Christensen,${ }^{92}$ P. Christiansen, ${ }^{83}$ T. Chujo, ${ }^{136}$ C. Cicalo, ${ }^{56}$ L. Cifarelli, ${ }^{26}$ F. Cindolo, ${ }^{55}$ M. R. Ciupek, ${ }^{110}$ G. Clai, ${ }^{55, b}$ J. Cleymans, ${ }^{126, a}$ F. Colamaria, ${ }^{54}$ J. S. Colburn, ${ }^{113}$ D. Colella, ${ }^{34,54,109,147}$ A. Collu, ${ }^{82}$ M. Colocci, ${ }^{26,35}$ M. Concas,${ }^{61, c}$ G. Conesa Balbastre ${ }^{81}$ Z. Conesa del Valle, ${ }^{80}$ G. Contin, ${ }^{24}$ J. G. Contreras, ${ }^{38}$ M. L. Coquet, ${ }^{140}$ T. M. Cormier, ${ }^{99}$ P. Cortese, ${ }^{32}$ M. R. Cosentino, ${ }^{125}$ F. Costa, ${ }^{35}$ S. Costanza, ${ }^{29,59}$ P. Crochet, ${ }^{137}$ E. Cuautle, ${ }^{71}$ P. Cui, ${ }^{7}$ L. Cunqueiro, ${ }^{99}$ A. Dainese, ${ }^{58}$ F. P. A. Damas, ${ }^{117,140}$ M. C. Danisch, ${ }^{107}$ A. Danu, ${ }^{69}$ I. Das, ${ }^{12}$ P. Das, ${ }^{89}$ P. Das, ${ }^{4}$ S. Das, ${ }^{4}$ S. Dash, ${ }^{50}$ S. De ${ }^{89}$ A. De Caro, ${ }^{30}$ G. de Cataldo,${ }^{54}$ L. De Cilladi, ${ }^{25}$ J. de Cuveland, ${ }^{40}$ A. De Falco, ${ }^{23}$ D. De Gruttola, ${ }^{30}$ N. De Marco, ${ }^{61}$ C. De Martin, ${ }^{24}$ S. De Pasquale, ${ }^{30}$ S. Deb,${ }^{51}$ H. F. Degenhardt, ${ }^{123}$ K. R. Deja, ${ }^{144}$ L. Dello Stritto, ${ }^{30}$ S. Delsanto, ${ }^{25}$ W. Deng, ${ }^{7}$ P. Dhankher, ${ }^{19}$ D. Di Bari, ${ }^{34}$ A. Di Mauro, ${ }^{35}$ R. A. Diaz, ${ }^{8}$ T. Dietel, ${ }^{126}$ Y. Ding, ${ }^{7,138}$ R. Divià, ${ }^{35}$ D. U. Dixit, ${ }^{19} \varnothing$. Djuvsland, ${ }^{21}$ U. Dmitrieva, ${ }^{65}$ J. Do, ${ }^{63}$ A. Dobrin, ${ }^{69}$ B. Dönigus, ${ }^{70}$ O. Dordic,${ }^{20}$ A. K. Dubey, ${ }^{143}$ A. Dubla, ${ }^{93,110}$ S. Dudi, ${ }^{103}$ M. Dukhishyam, ${ }^{89}$ P. Dupieux, ${ }^{137}$ N. Dzalaiova, ${ }^{13}$ T. M. Eder, ${ }^{146}$ R. J. Ehlers, ${ }^{99}$ V. N. Eikeland, ${ }^{21}$ D. Elia,${ }^{54}$ B. Erazmus, ${ }^{117}$ F. Ercolessi, ${ }^{26}$ F. Erhardt, ${ }^{102}$ A. Erokhin, ${ }^{115}$ M. R. Ersdal, ${ }^{21}$ B. Espagnon, ${ }^{80}$ G. Eulisse, ${ }^{35}$ D. Evans, ${ }^{113}$ S. Evdokimov, ${ }^{94}$ L. Fabbietti, ${ }^{108}$ M. Faggin, ${ }^{28}$ J. Faivre, ${ }^{81}$ F. Fan, ${ }^{7}$ A. Fantoni, ${ }^{53}$ M. Fasel, ${ }^{99}$ P. Fecchio, ${ }^{31}$ A. Feliciello, ${ }^{61}$ G. Feofilov, ${ }^{115}$ A. Fernández Téllez, ${ }^{46}$

A. Ferrero, ${ }^{140}$ A. Ferretti, ${ }^{25}$ V. J. G. Feuillard, ${ }^{107}$ J. Figiel, ${ }^{120}$ S. Filchagin, ${ }^{111}$ D. Finogeev, ${ }^{65}$ F. M. Fionda, ${ }^{21,56}$ G. Fiorenza, ${ }^{35,109}$ F. Flor, ${ }^{127}$ A. N. Flores, ${ }^{121}$ S. Foertsch, ${ }^{74}$ P. Foka, ${ }^{110}$ S. Fokin, ${ }^{91}$ E. Fragiacomo, ${ }^{62}$ E. Frajna, ${ }^{147}$ U. Fuchs,${ }^{35}$ N. Funicello, ${ }^{30}$ C. Furget, ${ }^{81}$ A. Furs, ${ }^{65}$ J. J. Gaardhøje, ${ }^{92}$ M. Gagliardi, ${ }^{25}$ A. M. Gago, ${ }^{114}$ A. Gal, ${ }^{139}$ C. D. Galvan, ${ }^{122}$ P. Ganoti ${ }^{87}$ C. Garabatos, ${ }^{110}$ J. R. A. Garcia, ${ }^{46}$ E. Garcia-Solis, ${ }^{10}$ K. Garg, ${ }^{117}$ C. Gargiulo, ${ }^{35}$ A. Garibli, ${ }^{90}$ K. Garner, ${ }^{146}$ P. Gasik, ${ }^{110}$ E. F. Gauger, ${ }^{121}$ A. Gautam, ${ }^{129}$ M. B. Gay Ducati, ${ }^{72}$ M. Germain, ${ }^{117}$ J. Ghosh ${ }^{112}$ P. Ghosh ${ }^{143}$ S. K. Ghosh, ${ }^{4}$ M. Giacalone ${ }^{26}$ P. Gianotti, ${ }^{53}$ P. Giubellino, ${ }^{61110}$ P. Giubilato, ${ }^{28}$ A. M. C. Glaenzer, ${ }^{140}$ P. Glässel, ${ }^{107}$ D. J. Q. Goh,${ }^{85}$ V. Gonzalez, ${ }^{145}$ L. H. González-Trueba, ${ }^{73}$ S. Gorbunov, ${ }^{40}$ M. Gorgon, ${ }^{2}$ L. Görlich, ${ }^{120}$ S. Gotovac, ${ }^{36}$ V. Grabski, ${ }^{73}$ L. K. Graczykowski, ${ }^{144}$ L. Greiner, ${ }^{82}$ A. Grelli, ${ }^{64}$ C. Grigoras ${ }^{35}$ V. Grigoriev,${ }^{96}$ A. Grigoryan, ${ }^{1, a}$ S. Grigoryan, ${ }^{1,77}$ O. S. Groettvik, ${ }^{21}$ F. Grosa, ${ }^{35,61}$ J. F. Grosse-Oetringhaus, ${ }^{35}$ R. Grosso, ${ }^{110}$ G. G. Guardiano, ${ }^{124}$ R. Guernane, ${ }^{81}$ M. Guilbaud,${ }^{17}$ K. Gulbrandsen, ${ }^{92}$ T. Gunji, ${ }^{135}$ A. Gupta, ${ }^{104}$ R. Gupta, ${ }^{104}$ S. P. Guzman, ${ }^{46}$ L. Gyulai, ${ }^{147}$ M. K. Habib, ${ }^{110}$ C. Hadjidakis, ${ }^{80}$ G. Halimoglu, ${ }^{70}$ H. Hamagaki, ${ }^{85}$ G. Hamar, ${ }^{147}$ M. Hamid, ${ }^{7}$ R. Hannigan, ${ }^{121}$ M. R. Haque,${ }^{89,144}$ A. Harlenderova, ${ }^{110}$ J. W. Harris, ${ }^{148}$ A. Harton, ${ }^{10}$ J. A. Hasenbichler, ${ }^{35}$ H. Hassan, ${ }^{99}$ D. Hatzifotiadou, ${ }^{55}$ P. Hauer, ${ }^{44}$ L. B. Havener, ${ }^{148}$ S. Hayashi, ${ }^{135}$ S. T. Heckel, ${ }^{108}$ E. Hellbär, ${ }^{70}$ H. Helstrup,${ }^{37}$ T. Herman, ${ }^{38}$ E. G. Hernandez, ${ }^{46}$ G. Herrera Corral, ${ }^{9}$ F. Herrmann, ${ }^{146}$ K. F. Hetland,${ }^{37}$ H. Hillemanns, ${ }^{35}$ C. Hills, ${ }^{130}$ B. Hippolyte, ${ }^{139}$ B. Hofman, ${ }^{64}$ B. Hohlweger, ${ }^{93,108}$ J. Honermann, ${ }^{146}$ G. H. Hong, ${ }^{149}$ D. Horak,${ }^{38}$ S. Hornung, ${ }^{110}$ A. Horzyk, ${ }^{2}$ R. Hosokawa, ${ }^{15}$ P. Hristov, ${ }^{35}$ C. Huang, ${ }^{80}$ C. Hughes, ${ }^{133}$ P. Huhn, ${ }^{70}$ T. J. Humanic, ${ }^{100}$ H. Hushnud, ${ }^{112}$ L. A. Husova, ${ }^{146}$ A. Hutson, ${ }^{127}$ D. Hutter, ${ }^{40}$ J. P. Iddon, ${ }^{35,130}$ R. Ilkaev, ${ }^{111}$ H. Ilyas, ${ }^{14}$ M. Inaba, ${ }^{136}$ G. M. Innocenti, ${ }^{35}$ M. Ippolitov, ${ }^{91}$ A. Isakov, ${ }^{38,98}$ M. S. Islam, ${ }^{112}$ 
M. Ivanov, ${ }^{110}$ V. Ivanov, ${ }^{101}$ V. Izucheev, ${ }^{94}$ M. Jablonski, ${ }^{2}$ B. Jacak, ${ }^{82}$ N. Jacazio,${ }^{35}$ P. M. Jacobs, ${ }^{82}$ S. Jadlovska, ${ }^{119}$ J. Jadlovsky, ${ }^{119}$ S. Jaelani, ${ }^{64}$ C. Jahnke, ${ }^{123,124}$ M. J. Jakubowska, ${ }^{144}$ A. Jalotra, ${ }^{104}$ M. A. Janik, ${ }^{144}$ T. Janson, ${ }^{76}$ M. Jercic, ${ }^{102}$ O. Jevons, ${ }^{113}$ F. Jonas,,${ }^{99,146}$ P. G. Jones, ${ }^{113}$ J. M. Jowett, ${ }^{35,110}$ J. Jung, ${ }^{70}$ M. Jung, ${ }^{70}$ A. Junique, ${ }^{35}$ A. Jusko, ${ }^{113}$ J. Kaewjai, ${ }^{118}$ P. Kalinak, ${ }^{66}$ A. Kalweit, ${ }^{35}$ V. Kaplin, ${ }^{96}$ S. Kar, ${ }^{7}$ A. Karasu Uysal, ${ }^{79}$ D. Karatovic, ${ }^{102}$ O. Karavichev, ${ }^{65}$ T. Karavicheva, ${ }^{65}$ P. Karczmarczyk, ${ }^{144}$ E. Karpechev, ${ }^{65}$ A. Kazantsev, ${ }^{91}$ U. Kebschull, ${ }^{76}$ R. Keidel, ${ }^{48}$ D. L. D. Keijdener, ${ }^{64}$ M. Keil, ${ }^{35}$

B. Ketzer, ${ }^{44}$ Z. Khabanova, ${ }^{93}$ A. M. Khan, ${ }^{7}$ S. Khan, ${ }^{16}$ A. Khanzadeev, ${ }^{101}$ Y. Kharlov, ${ }^{94}$ A. Khatun, ${ }^{16}$ A. Khuntia, ${ }^{120}$ B. Kileng, ${ }^{37}$ B. Kim, ${ }^{17,63}$ C. Kim, ${ }^{17}$ D. Kim, ${ }^{149}$ D. J. Kim, ${ }^{128}$ E. J. Kim, ${ }^{75}$ J. Kim, ${ }^{149}$ J. S. Kim, ${ }^{42}$ J. Kim, ${ }^{107}$ J. Kim, ${ }^{149}$ J. Kim, ${ }^{75}$ M. Kim, ${ }^{107}$ S. Kim, ${ }^{18}$ T. Kim, ${ }^{149}$ S. Kirsch,${ }^{70}$ I. Kisel, ${ }^{40}$ S. Kiselev, ${ }^{95}$ A. Kisiel, ${ }^{144}$ J. P. Kitowski, ${ }^{2}$ J. L. Klay, ${ }^{6}$ J. Klein, ${ }^{35}$ S. Klein, ${ }^{82}$ C. Klein-Bösing, ${ }^{146}$ M. Kleiner, ${ }^{70}$ T. Klemenz, ${ }^{108}$ A. Kluge, ${ }^{35}$ A. G. Knospe, ${ }^{127}$ C. Kobdaj, ${ }^{118}$ M. K. Köhler, ${ }^{107}$ T. Kollegger, ${ }^{110}$ A. Kondratyev, ${ }^{77}$ N. Kondratyeva, ${ }^{96}$ E. Kondratyuk, ${ }^{94}$ J. Konig, ${ }^{70}$ S. A. Konigstorfer, ${ }^{108}$ P. J. Konopka, ${ }^{2,35}$ G. Kornakov, ${ }^{144}$ S. D. Koryciak, ${ }^{2}$ L. Koska, ${ }^{119}$ A. Kotliarov, ${ }^{98}$ O. Kovalenko, ${ }^{88}$ V. Kovalenko, ${ }^{115}$ M. Kowalski, ${ }^{120}$ I. Králik, ${ }^{66}$ A. Kravčáková, ${ }^{39}$ L. Kreis, ${ }^{110}$ M. Krivda, ${ }^{66,113}$ F. Krizek, ${ }^{98}$ K. Krizkova Gajdosova ${ }^{38}$ M. Kroesen, ${ }^{107}$ M. Krüger, ${ }^{70}$ E. Kryshen, ${ }^{101}$ M. Krzewicki, ${ }^{40}$ V. Kučera ${ }^{35}$ C. Kuhn, ${ }^{139}$ P. G. Kuijer, ${ }^{93}$ T. Kumaoka, ${ }^{136}$ D. Kumar, ${ }^{143}$ L. Kumar, ${ }^{103}$ N. Kumar, ${ }^{103}$ S. Kundu, ${ }^{35,89}$ P. Kurashvili, ${ }^{88}$ A. Kurepin, ${ }^{65}$ A. B. Kurepin,${ }^{65}$ A. Kuryakin, ${ }^{111}$ S. Kushpil, ${ }^{98}$ J. Kvapil, ${ }^{113}$ M. J. Kweon, ${ }^{63}$ J. Y. Kwon, ${ }^{63}$ Y. Kwon, ${ }^{149}$ S. L. La Pointe, ${ }^{40}$ P. La Rocca, ${ }^{27}$ Y. S. Lai, ${ }^{82}$ A. Lakrathok, ${ }^{118}$ M. Lamanna,${ }^{35}$ R. Langoy, ${ }^{132}$ K. Lapidus,${ }^{35}$ P. Larionov, ${ }^{53}$ E. Laudi, ${ }^{35}$ L. Lautner, ${ }^{35,108}$ R. Lavicka ${ }^{38}$ T. Lazareva, ${ }^{115}$ R. Lea,${ }^{24,59,142}$ J. Lehrbach, ${ }^{40}$ R. C. Lemmon, ${ }^{97}$ I. León Monzón, ${ }^{122}$ E. D. Lesser, ${ }^{19}$ M. Lettrich, ${ }^{35,108}$ P. Lévai, ${ }^{147}$ X. Li, ${ }^{11}$ X. L. Li, ${ }^{7}$ J. Lien, ${ }^{132}$ R. Lietava, ${ }^{113}$ B. Lim, ${ }^{17}$ S. H. Lim,${ }^{17}$ V. Lindenstruth,,${ }^{40}$ A. Lindner, ${ }^{49}$ C. Lippmann, ${ }^{110}$ A. Liu, ${ }^{19}$ J. Liu, ${ }^{130}$ I. M. Lofnes,${ }^{21}$ V. Loginov,${ }^{96}$ C. Loizides, ${ }^{99}$ P. Loncar,${ }^{36}$ J. A. Lopez, ${ }^{107}$ X. Lopez, ${ }^{137}$ E. López Torres, ${ }^{8}$ J. R. Luhder, ${ }^{146}$ M. Lunardon, ${ }^{28}$ G. Luparello, ${ }^{62}$ Y. G. Ma, ${ }^{41}$ A. Maevskaya, ${ }^{65}$ M. Mager,${ }^{35}$ T. Mahmoud,${ }^{44}$ A. Maire, ${ }^{139}$ M. Malaev, ${ }^{101}$ N. M. Malik, ${ }^{104}$ Q. W. Malik, ${ }^{20}$ L. Malinina, ${ }^{77, d}$ D. Mal'Kevich, ${ }^{95}$ N. Mallick,${ }^{51}$ P. Malzacher, ${ }^{110}$ G. Mandaglio, ${ }^{33,57}$ V. Manko, ${ }^{91}$ F. Manso, ${ }^{137}$ V. Manzari, ${ }^{54}$ Y. Mao, ${ }^{7}$ J. Mareš, ${ }^{68}$ G. V. Margagliotti,${ }^{24}$ A. Margotti,${ }^{55}$

A. Marín, ${ }^{10}$ C. Markert, ${ }^{121}$ M. Marquard,${ }^{70}$ N. A. Martin, ${ }^{107}$ P. Martinengo, ${ }^{35}$ J. L. Martinez, ${ }^{127}$ M. I. Martínez, ${ }^{46}$ G. Martínez García, ${ }^{17}$ S. Masciocchi, ${ }^{10}$ M. Masera, ${ }^{25}$ A. Masoni, ${ }^{56}$ L. Massacrier, ${ }^{80}$ A. Mastroserio,${ }^{54,141}$ A. M. Mathis, ${ }^{108}$ O. Matonoha, ${ }^{83}$ P. F. T. Matuoka, ${ }^{123}$ A. Matyja,${ }^{120}$ C. Mayer,${ }^{120}$ A. L. Mazuecos, ${ }^{35}$ F. Mazzaschi, ${ }^{25}$ M. Mazzilli, ${ }^{35}$ M. A. Mazzoni, ${ }^{60}$ J. E. Mdhluli, ${ }^{134}$ A. F. Mechler, ${ }^{70}$ F. Meddi, ${ }^{22}$ Y. Melikyan, ${ }^{65}$ A. Menchaca-Rocha, ${ }^{73}$ E. Meninno, ${ }^{30,116}$ A. S. Menon, ${ }^{127}$ M. Meres, ${ }^{13}$ S. Mhlanga, ${ }^{74,126}$ Y. Miake, ${ }^{136}$ L. Micheletti, ${ }^{25,61}$ L. C. Migliorin,${ }^{138}$ D. L. Mihaylov, ${ }^{108}$ K. Mikhaylov ${ }^{77,95}$ A. N. Mishra, ${ }^{147}$ D. Miśkowiec, ${ }^{110}$ A. Modak, ${ }^{4}$ A. P. Mohanty, ${ }^{64}$ B. Mohanty, ${ }^{89}$ M. Mohisin Khan, ${ }^{16}$ Z. Moravcova, ${ }^{92}$ C. Mordasini, ${ }^{108}$ D. A. Moreira De Godoy, ${ }^{146}$ L. A. P. Moreno, ${ }^{46}$ I. Morozov, ${ }^{65}$ A. Morsch, ${ }^{35}$ T. Mrnjavac, ${ }^{35}$ V. Muccifora ${ }^{53}$ E. Mudnic, ${ }^{36}$ D. Mühlheim ${ }^{146}$ S. Muhuri, ${ }^{143}$ J. D. Mulligan, ${ }^{82}$ A. Mulliri, ${ }^{23}$ M. G. Munhoz, ${ }^{123}$ R. H. Munzer, ${ }^{70}$ H. Murakami, ${ }^{135}$ S. Murray, ${ }^{126}$ L. Musa, ${ }^{35}$ J. Musinsky, ${ }^{66}$ J. W. Myrcha, ${ }^{144}$ B. Naik, ${ }^{50,134}$ R. Nair, ${ }^{88}$ B. K. Nandi, ${ }^{50}$ R. Nania,${ }^{55}$ E. Nappi,${ }^{54}$ M. U. Naru, ${ }^{14}$ A. F. Nassirpour, ${ }^{83}$ A. Nath, ${ }^{107}$ C. Nattrass, ${ }^{133}$ A. Neagu, ${ }^{20}$ L. Nellen, ${ }^{71}$ S. V. Nesbo, ${ }^{37}$ G. Neskovic, ${ }^{40}$ D. Nesterov, ${ }^{115}$ B. S. Nielsen, ${ }^{92}$ S. Nikolaev, ${ }^{91}$ S. Nikulin, ${ }^{91}$ V. Nikulin, ${ }^{101}$ F. Noferini, ${ }^{55}$ S. Noh, ${ }^{12}$ P. Nomokonov, ${ }^{77}$ J. Norman, ${ }^{130}$ N. Novitzky, ${ }^{136}$ P. Nowakowski, ${ }^{144}$ A. Nyanin, ${ }^{91}$ J. Nystrand,${ }^{21}$ M. Ogino, ${ }^{85}$ A. Ohlson, ${ }^{83}$ V. A. Okorokov, ${ }^{96}$ J. Oleniacz, ${ }^{144}$ A. C. Oliveira Da Silva, ${ }^{133}$ M. H. Oliver, ${ }^{148}$ A. Onnerstad, ${ }^{128}$

C. Oppedisano, ${ }^{61}$ A. Ortiz Velasquez, ${ }^{71}$ T. Osako, ${ }^{47}$ A. Oskarsson, ${ }^{83}$ J. Otwinowski, ${ }^{120}$ K. Oyama, ${ }^{85}$ Y. Pachmayer,${ }^{107}$ S. Padhan,${ }^{50}$ D. Pagano, ${ }^{59,142}$ G. Paić, ${ }^{71}$ A. Palasciano, ${ }^{54}$ J. Pan, ${ }^{145}$ S. Panebianco, ${ }^{140}$ P. Pareek,${ }^{143}$ J. Park, ${ }^{63}$ J. E. Parkkila, ${ }^{128}$

S. P. Pathak, ${ }^{127}$ R. N. Patra ${ }^{35,104}$ B. Paul, ${ }^{23}$ J. Pazzini, ${ }^{59,142}$ H. Pei, ${ }^{7}$ T. Peitzmann, ${ }^{64}$ X. Peng, ${ }^{7}$ L. G. Pereira, ${ }^{72}$

H. Pereira Da Costa, ${ }^{140}$ D. Peresunko, ${ }^{91}$ G. M. Perez, ${ }^{8}$ S. Perrin, ${ }^{140}$ Y. Pestov, ${ }^{5}$ V. Petráček, ${ }^{38}$ M. Petrovici, ${ }^{49}$ R. P. Pezzi, ${ }^{72}$ S. Piano, ${ }^{62}$ M. Pikna, ${ }^{13}$ P. Pillot, ${ }^{117}$ O. Pinazza, ${ }^{35,55}$ L. Pinsky, ${ }^{127}$ C. Pinto, ${ }^{27}$ S. Pisano, ${ }^{53}$ M. Płoskoń, ${ }^{82}$ M. Planinic, ${ }^{102}$ F. Pliquett, ${ }^{70}$ M. G. Poghosyan, ${ }^{99}$ B. Polichtchouk, ${ }^{94}$ S. Politano, ${ }^{31}$ N. Poljak, ${ }^{102}$ A. Pop, ${ }^{49}$ S. Porteboeuf-Houssais, ${ }^{137}$ J. Porter, ${ }^{82}$ V. Pozdniakov, ${ }^{77}$ S. K. Prasad, ${ }^{4}$ R. Preghenella, ${ }^{55}$ F. Prino, ${ }^{61}$ C. A. Pruneau, ${ }^{145}$ I. Pshenichnov, ${ }^{65}$ M. Puccio, ${ }^{35}$ S. Qiu, ${ }^{93}$ L. Quaglia, ${ }^{25}$ R. E. Quishpe, ${ }^{127}$ S. Ragoni, ${ }^{113}$ A. Rakotozafindrabe, ${ }^{140}$ L. Ramello, ${ }^{32}$ F. Rami, ${ }^{139}$

S. A. R. Ramirez, ${ }^{46}$ A. G. T. Ramos, ${ }^{34}$ T. A. Rancien, ${ }^{81}$ R. Raniwala, ${ }^{105}$ S. Raniwala, ${ }^{105}$ S. S. Räsänen, ${ }^{45}$ R. Rath ${ }^{51}$ I. Ravasenga, ${ }^{93}$ K. F. Read ${ }^{99,133}$ A. R. Redelbach, ${ }^{40}$ K. Redlich, ${ }^{88, e}$ A. Rehman, ${ }^{21}$ P. Reichelt, ${ }^{70}$ F. Reidt, ${ }^{35}$

H. A. Reme-ness, ${ }^{37}$ R. Renfordt, ${ }^{70}$ Z. Rescakova, ${ }^{39}$ K. Reygers, ${ }^{107}$ A. Riabov, ${ }^{101}$ V. Riabov, ${ }^{101}$ T. Richert, ${ }^{83,92}$ M. Richter, ${ }^{20}$ W. Riegler, ${ }^{35}$ F. Riggi, ${ }^{27}$ C. Ristea ${ }^{69}$ S. P. Rode,${ }^{51}$ M. Rodríguez Cahuantzi, ${ }^{46}$ K. Røed,${ }^{20}$ R. Rogalev, ${ }^{94}$ E. Rogochaya, ${ }^{77}$ T. S. Rogoschinski, ${ }^{70}$ D. Rohr, ${ }^{35}$ D. Röhrich,${ }^{21}$ P. F. Rojas, ${ }^{46}$ P. S. Rokita,${ }^{144}$ F. Ronchetti, ${ }^{53}$ A. Rosano, ${ }^{33,57}$ E. D. Rosas,${ }^{71}$ A. Rossi, ${ }^{58}$ A. Rotondi, ${ }^{29,59}$ A. Roy, ${ }^{51}$ P. Roy, ${ }^{112}$ S. Roy, ${ }^{50}$ N. Rubini, ${ }^{26}$ O. V. Rueda, ${ }^{83}$ R. Rui, ${ }^{24}$ B. Rumyantsev,${ }^{77}$ 
P. G. Russek, ${ }^{2}$ A. Rustamov, ${ }^{90}$ E. Ryabinkin, ${ }^{91}$ Y. Ryabov, ${ }^{101}$ A. Rybicki, ${ }^{120}$ H. Rytkonen, ${ }^{128}$ W. Rzesa, ${ }^{144}$ O. A. M. Saarimaki,${ }^{45}$ R. Sadek, ${ }^{117}$ S. Sadovsky, ${ }^{94}$ J. Saetre, ${ }^{21}$ K. Šafařík ${ }^{38}$ S. K. Saha, ${ }^{143}$ S. Saha, ${ }^{89}$ B. Sahoo, ${ }^{50}$ P. Sahoo,${ }^{50}$ R. Sahoo, ${ }^{51}$ S. Sahoo, ${ }^{67}$ D. Sahu, ${ }^{51}$ P. K. Sahu, ${ }^{67}$ J. Saini, ${ }^{143}$ S. Sakai, ${ }^{136}$ S. Sambyal, ${ }^{104}$ V. Samsonov,${ }^{96,101, a}$ D. Sarkar, ${ }^{145}$ N. Sarkar, ${ }^{143}$ P. Sarma, ${ }^{43}$ V. M. Sarti, ${ }^{108}$ M. H. P. Sas, ${ }^{148}$ J. Schambach, ${ }^{99,121}$ H. S. Scheid,${ }^{70}$ C. Schiaua,${ }^{49}$ R. Schicker, ${ }^{107}$ A. Schmah, ${ }^{107}$ C. Schmidt, ${ }^{110}$ H. R. Schmidt, ${ }^{106}$ M. O. Schmidt, ${ }^{107}$ M. Schmidt, ${ }^{106}$ N. V. Schmidt, ${ }^{70,99}$ A. R. Schmier, ${ }^{133}$ R. Schotter, ${ }^{139}$ J. Schukraft, ${ }^{35}$ Y. Schutz, ${ }^{139}$ K. Schwarz, ${ }^{110}$ K. Schweda, ${ }^{110}$ G. Scioli, ${ }^{26}$ E. Scomparin, ${ }^{61}$ J. E. Seger, ${ }^{15}$ Y. Sekiguchi, ${ }^{135}$ D. Sekihata, ${ }^{135}$ I. Selyuzhenkov, ${ }^{96,110}$ S. Senyukov, ${ }^{139}$ J. J. Seo,${ }^{63}$ D. Serebryakov, ${ }^{65}$ L. Šerkšnytė,${ }^{108}$ A. Sevcenco, ${ }^{69}$ T. J. Shaba, ${ }^{74}$ A. Shabanov, ${ }^{65}$ A. Shabetai, ${ }^{117}$ R. Shahoyan, ${ }^{35}$ W. Shaikh, ${ }^{112}$ A. Shangaraev, ${ }^{94}$ A. Sharma, ${ }^{103}$ H. Sharma, ${ }^{120}$ M. Sharma, ${ }^{104}$ N. Sharma, ${ }^{103}$ S. Sharma, ${ }^{104}$ U. Sharma, ${ }^{104}$ O. Sheibani, ${ }^{127}$ K. Shigaki, ${ }^{47}$ M. Shimomura, ${ }^{86}$ S. Shirinkin, ${ }^{95}$ Q. Shou, ${ }^{41}$ Y. Sibiriak, ${ }^{91}$ S. Siddhanta, ${ }^{56}$ T. Siemiarczuk, ${ }^{88}$ T. F. Silva, ${ }^{123}$ D. Silvermyr, ${ }^{83}$ G. Simonetti, ${ }^{35}$ B. Singh, ${ }^{108}$ R. Singh, ${ }^{89}$ R. Singh,${ }^{104}$ R. Singh,${ }^{51}$ V. K. Singh, ${ }^{143}$ V. Singhal, ${ }^{143}$ T. Sinha, ${ }^{112}$ B. Sitar,${ }^{13}$ M. Sitta, ${ }^{32}$ T. B. Skaali, ${ }^{20}$ G. Skorodumovs, ${ }^{107}$ M. Slupecki, ${ }^{45}$ N. Smirnov, ${ }^{148}$ R. J. M. Snellings, ${ }^{64}$ C. Soncco, ${ }^{114}$ J. Song, ${ }^{127}$ A. Songmoolnak, ${ }^{18}$ F. Soramel, ${ }^{28}$ S. Sorensen, ${ }^{133}$ I. Sputowska, ${ }^{120}$ J. Stachel, ${ }^{107}$ I. Stan, ${ }^{69}$ P. J. Steffanic, ${ }^{133}$ S. F. Stiefelmaier, ${ }^{107}$ D. Stocco, ${ }^{17}$ I. Storehaug, ${ }^{20}$ M. M. Storetvedt, ${ }^{37}$ C. P. Stylianidis, ${ }^{93}$ A. A. P. Suaide, ${ }^{123}$ T. Sugitate,${ }^{47}$ C. Suire ${ }^{80}$ M. Suljic, ${ }^{35}$ R. Sultanov,${ }^{95}$ M. Šumbera, ${ }^{98}$ V. Sumberia, ${ }^{104}$ S. Sumowidagdo, ${ }^{52}$ S. Swain, ${ }^{67}$ A. Szabo, ${ }^{13}$ I. Szarka, ${ }^{13}$ U. Tabassam, ${ }^{14}$ S. F. Taghavi, ${ }^{108}$ G. Taillepied, ${ }^{137}$ J. Takahashi, ${ }^{124}$ G. J. Tambave, ${ }^{21}$ S. Tang, ${ }^{7,137}$ Z. Tang, ${ }^{131}$ M. Tarhini,${ }^{117}$ M. G. Tarzila, ${ }^{49}$ A. Tauro, ${ }^{35}$ G. Tejeda Muñoz, ${ }^{46}$ A. Telesca, ${ }^{35}$ L. Terlizzi, ${ }^{25}$ C. Terrevoli, ${ }^{127}$ G. Tersimonov, ${ }^{3}$ S. Thakur, ${ }^{143}$ D. Thomas, ${ }^{121}$ R. Tieulent, ${ }^{138}$ A. Tikhonov, ${ }^{65}$ A. R. Timmins, ${ }^{127}$ M. Tkacik, ${ }^{119}$ A. Toia, ${ }^{70}$ N. Topilskaya,${ }^{65}$ M. Toppi,${ }^{53}$ F. Torales-Acosta, ${ }^{19}$ T. Tork,${ }^{80}$ R. C. Torres, ${ }^{82}$ S. R. Torres,${ }^{38}$ A. Trifiro,,${ }^{33,57}$ S. Tripathy, ${ }^{55,71}$ T. Tripathy,${ }^{50}$ S. Trogolo, ${ }^{28,35}$ G. Trombetta, ${ }^{34}$ V. Trubnikov, ${ }^{3}$ W. H. Trzaska, ${ }^{128}$ T. P. Trzcinski, ${ }^{144}$ B. A. Trzeciak, ${ }^{38}$ A. Tumkin, ${ }^{111}$ R. Turrisi, ${ }^{58}$ T. S. Tveter, ${ }^{20}$ K. Ullaland, ${ }^{21}$ A. Uras, ${ }^{138}$ M. Urioni,,${ }^{59,142}$ G. L. Usai, ${ }^{23}$ M. Vala, ${ }^{39}$ N. Valle,${ }^{29,59}$ S. Vallero, ${ }^{61}$ N. van der Kolk ${ }^{64}$ L. V. R. van Doremalen, ${ }^{64}$ M. van Leeuwen, ${ }^{93}$ P. Vande Vyvre, ${ }^{35}$ D. Varga, ${ }^{147}$ Z. Varga, ${ }^{147}$ M. Varga-Kofarago, ${ }^{147}$ A. Vargas,${ }^{46}$ M. Vasileiou, ${ }^{87}$ A. Vasiliev,${ }^{91}$ O. Vázquez Doce, ${ }^{108}$ V. Vechernin,,${ }^{115}$ E. Vercellin, ${ }^{25}$ S. Vergara Limón, ${ }^{46}$ L. Vermunt, ${ }^{64}$ R. Vértesi, ${ }^{147}$ M. Verweij,${ }^{64}$ L. Vickovic,${ }^{36}$ Z. Vilakazi,${ }^{134}$ O. Villalobos Baillie, ${ }^{113}$ G. Vino, ${ }^{54}$ A. Vinogradov, ${ }^{91}$ T. Virgili, ${ }^{30}$ V. Vislavicius, ${ }^{92}$ A. Vodopyanov, ${ }^{77}$ B. Volkel, ${ }^{35}$ M. A. Völkl, ${ }^{107}$ K. Voloshin, ${ }^{95}$ S. A. Voloshin, ${ }^{145}$ G. Volpe, ${ }^{34}$ B. von Haller, ${ }^{35}$ I. Vorobyev, ${ }^{108}$ D. Voscek, ${ }^{119}$ N. Vozniuk, ${ }^{65}$ J. Vrláková, ${ }^{39}$ B. Wagner, ${ }^{21}$ C. Wang, ${ }^{41}$ D. Wang, ${ }^{41}$ M. Weber, ${ }^{116}$ R. J. G. V. Weelden, ${ }^{93}$ A. Wegrzynek, ${ }^{35}$ S. C. Wenzel, ${ }^{35}$ J. P. Wessels,${ }^{146}$ J. Wiechula, ${ }^{70}$ J. Wikne, ${ }^{20}$ G. Wilk, ${ }^{88}$ J. Wilkinson, ${ }^{110}$ G. A. Willems, ${ }^{146}$ B. Windelband, ${ }^{107}$ M. Winn, ${ }^{140}$ W. E. Witt, ${ }^{133}$ J. R. Wright, ${ }^{121}$ W. Wu, ${ }^{41}$ Y. Wu, ${ }^{131}$ R. Xu, ${ }^{7}$ S. Yalcin, ${ }^{79}$ Y. Yamaguchi,${ }^{47}$ K. Yamakawa, ${ }^{47}$ S. Yang, ${ }^{21}$ S. Yano, ${ }^{47}$ Z. Yin, ${ }^{7}$ H. Yokoyama,${ }^{64}$ I.-K. Yoo, ${ }^{17}$ J. H. Yoon, ${ }^{63}$ S. Yuan, ${ }^{21}$ A. Yuncu, ${ }^{107}$ V. Zaccolo, ${ }^{24}$ A. Zaman, ${ }^{14}$ C. Zampolli, ${ }^{35}$ H. J. C. Zanoli, ${ }^{64}$ N. Zardoshti ${ }^{35}$ A. Zarochentsev, ${ }^{115}$ P. Závada, ${ }^{68}$ N. Zaviyalov, ${ }^{111}$ H. Zbroszczyk, ${ }^{144}$ M. Zhalov, ${ }^{101}$ S. Zhang, ${ }^{41}$ X. Zhang, ${ }^{7}$ Y. Zhang, ${ }^{131}$ V. Zherebchevskii, ${ }^{115}$ Y. Zhi, ${ }^{11}$ D. Zhou, ${ }^{7}$ Y. Zhou, ${ }^{92}$ J. Zhu, ${ }^{7,110}$ Y. Zhu, ${ }^{7}$ A. Zichichi, ${ }^{26}$ G. Zinovjev, ${ }^{3}$ and N. Zurlo ${ }^{59,142}$

\title{
(ALICE Collaboration)
}

\author{
${ }^{1}$ A.I. Alikhanyan National Science Laboratory (Yerevan Physics Institute) Foundation, Yerevan, Armenia \\ ${ }^{2}$ AGH University of Science and Technology, Cracow, Poland \\ ${ }^{3}$ Bogolyubov Institute for Theoretical Physics, National Academy of Sciences of Ukraine, Kiev, Ukraine \\ ${ }^{4}$ Bose Institute, Department of Physics and Centre for Astroparticle Physics and Space Science (CAPSS), \\ Kolkata, India \\ ${ }^{5}$ Budker Institute for Nuclear Physics, Novosibirsk, Russia \\ ${ }^{6}$ California Polytechnic State University, San Luis Obispo, California, USA \\ ${ }^{7}$ Central China Normal University, Wuhan, China \\ ${ }^{8}$ Centro de Aplicaciones Tecnológicas y Desarrollo Nuclear (CEADEN), Havana, Cuba \\ ${ }^{9}$ Centro de Investigación y de Estudios Avanzados (CINVESTAV), Mexico City and Mérida, Mexico \\ ${ }^{10}$ Chicago State University, Chicago, Illinois, USA \\ ${ }^{11}$ China Institute of Atomic Energy, Beijing, China \\ ${ }^{12}$ Chungbuk National University, Cheongju, Republic of Korea \\ ${ }^{13}$ Comenius University Bratislava, Faculty of Mathematics, Physics and Informatics, Bratislava, Slovakia \\ ${ }^{14}$ COMSATS University Islamabad, Islamabad, Pakistan \\ ${ }^{15}$ Creighton University, Omaha, Nebraska, USA \\ ${ }^{16}$ Department of Physics, Aligarh Muslim University, Aligarh, India
}


${ }^{17}$ Department of Physics, Pusan National University, Pusan, Republic of Korea

${ }^{18}$ Department of Physics, Sejong University, Seoul, Republic of Korea

${ }^{19}$ Department of Physics, University of California, Berkeley, California, USA

${ }^{20}$ Department of Physics, University of Oslo, Oslo, Norway

${ }^{21}$ Department of Physics and Technology, University of Bergen, Bergen, Norway

${ }^{22}$ Dipartimento di Fisica dell'Università 'La Sapienza'and Sezione INFN, Rome, Italy

${ }^{23}$ Dipartimento di Fisica dell'Università, and Sezione INFN, Cagliari, Italy

${ }^{24}$ Dipartimento di Fisica dell'Università, and Sezione INFN, Trieste, Italy

${ }^{25}$ Dipartimento di Fisica dell'Università, and Sezione INFN, Turin, Italy

${ }^{26}$ Dipartimento di Fisica e Astronomia dell'Università, and Sezione INFN, Bologna, Italy

${ }^{27}$ Dipartimento di Fisica e Astronomia dell'Università, and Sezione INFN, Catania, Italy

${ }^{28}$ Dipartimento di Fisica e Astronomia dell'Università, and Sezione INFN, Padova, Italy

${ }^{29}$ Dipartimento di Fisica e Nucleare e Teorica, Università di Pavia, Pavia, Italy

${ }^{30}$ Dipartimento di Fisica 'E.R. Caianiello' dell'Università, and Gruppo Collegato INFN, Salerno, Italy

${ }^{31}$ Dipartimento DISAT del Politecnico and Sezione INFN, Turin, Italy

${ }^{32}$ Dipartimento di Scienze e Innovazione Tecnologica dell'Università del Piemonte Orientale and INFN Sezione di Torino, Alessandria, Italy

${ }^{33}$ Dipartimento di Scienze MIFT, Università di Messina, Messina, Italy

${ }^{34}$ Dipartimento Interateneo di Fisica 'M. Merlin', and Sezione INFN, Bari, Italy

${ }^{35}$ European Organization for Nuclear Research (CERN), Geneva, Switzerland

${ }^{36}$ Faculty of Electrical Engineering, Mechanical Engineering and Naval Architecture, University of Split, Split, Croatia

${ }^{37}$ Faculty of Engineering and Science, Western Norway University of Applied Sciences, Bergen, Norway

${ }^{38}$ Faculty of Nuclear Sciences and Physical Engineering, Czech Technical University in Prague,

Prague, Czech Republic

${ }^{39}$ Faculty of Science, P.J. Šafárik University, Košice, Slovakia

${ }^{40}$ Frankfurt Institute for Advanced Studies, Johann Wolfgang Goethe-Universität Frankfurt,

Frankfurt, Germany

${ }^{41}$ Fudan University, Shanghai, China

${ }^{42}$ Gangneung-Wonju National University, Gangneung, Republic of Korea

${ }^{43}$ Gauhati University, Department of Physics, Guwahati, India

${ }^{44}$ Helmholtz-Institut für Strahlen- und Kernphysik, Rheinische Friedrich-Wilhelms-Universität Bonn, Bonn, Germany

${ }^{45}$ Helsinki Institute of Physics (HIP), Helsinki, Finland

${ }^{46}$ High Energy Physics Group, Universidad Autónoma de Puebla, Puebla, Mexico

${ }^{47}$ Hiroshima University, Hiroshima, Japan

${ }^{48}$ Hochschule Worms, Zentrum für Technologietransfer und Telekommunikation (ZTT), Worms, Germany

${ }^{49}$ Horia Hulubei National Institute of Physics and Nuclear Engineering, Bucharest, Romania

${ }^{50}$ Indian Institute of Technology Bombay (IIT), Mumbai, India

${ }^{51}$ Indian Institute of Technology Indore, Indore, India

${ }^{52}$ Indonesian Institute of Sciences, Jakarta, Indonesia

${ }^{53}$ INFN, Laboratori Nazionali di Frascati, Frascati, Italy

${ }^{54}$ INFN, Sezione di Bari, Bari, Italy

${ }^{55}$ INFN, Sezione di Bologna, Bologna, Italy

${ }^{56}$ INFN, Sezione di Cagliari, Cagliari, Italy

${ }^{57}$ INFN, Sezione di Catania, Catania, Italy

${ }^{58}$ INFN, Sezione di Padova, Padova, Italy

${ }^{59}$ INFN, Sezione di Pavia, Pavia, Italy

${ }^{60}$ INFN, Sezione di Roma, Rome, Italy

${ }^{61}$ INFN, Sezione di Torino, Turin, Italy

${ }^{62}$ INFN, Sezione di Trieste, Trieste, Italy

${ }^{63}$ Inha University, Republic of Korea

${ }^{64}$ Institute for Gravitational and Subatomic Physics (GRASP), Utrecht University/Nikhef, Utrecht, Netherlands

${ }^{65}$ Institute for Nuclear Research, Academy of Sciences, Moscow, Russia

${ }^{66}$ Institute of Experimental Physics, Slovak Academy of Sciences, Košice, Slovakia

${ }^{67}$ Institute of Physics, Homi Bhabha National Institute, Bhubaneswar, India

${ }^{68}$ Institute of Physics of the Czech Academy of Sciences, Prague, Czech Republic

${ }^{69}$ Institute of Space Science (ISS), Bucharest, Romania

${ }^{70}$ Institut für Kernphysik, Johann Wolfgang Goethe-Universität Frankfurt, Frankfurt, Germany 
${ }^{71}$ Instituto de Ciencias Nucleares, Universidad Nacional Autónoma de México, Mexico City, Mexico

${ }^{72}$ Instituto de Física, Universidade Federal do Rio Grande do Sul (UFRGS), Porto Alegre, Brazil

${ }^{73}$ Instituto de Física, Universidad Nacional Autónoma de México, Mexico City, Mexico

${ }^{74}$ iThemba LABS, National Research Foundation, Somerset West, South Africa

${ }^{75}$ Jeonbuk National University, Jeonju, Republic of Korea

${ }^{76}$ Johann-Wolfgang-Goethe Universität Frankfurt Institut für Informatik, Fachbereich Informatik und Mathematik, Frankfurt, Germany

${ }^{77}$ Joint Institute for Nuclear Research (JINR), Dubna, Russia

${ }^{78}$ Korea Institute of Science and Technology Information, Daejeon, Republic of Korea

${ }^{79}$ KTO Karatay University, Konya, Turkey

${ }^{80}$ Laboratoire de Physique des 2 Infinis, Irène Joliot-Curie, Irène Joliot-Curie, Orsay, France

${ }^{81}$ Laboratoire de Physique Subatomique et de Cosmologie, Université Grenoble-Alpes, CNRS-IN2P3, Grenoble, France

${ }^{82}$ Lawrence Berkeley National Laboratory, California, USA

${ }^{83}$ Lund University Department of Physics, Division of Particle Physics, Lund, Sweden

${ }^{84}$ Moscow Institute for Physics and Technology, Moscow, Russia

${ }^{85}$ Nagasaki Institute of Applied Science, Nagasaki, Japan

${ }^{86}$ Nara Women's University (NWU), Nara, Japan

${ }^{87}$ National and Kapodistrian University of Athens, School of Science, Department of Physics, Athens, Greece

${ }^{88}$ National Centre for Nuclear Research, Warsaw, Poland

${ }^{89}$ National Institute of Science Education and Research, Homi Bhabha National Institute, Jatni, India

${ }^{90}$ National Nuclear Research Center, Baku, Azerbaijan

${ }^{91}$ National Research Centre Kurchatov Institute, Moscow, Russia

${ }^{92}$ Niels Bohr Institute, University of Copenhagen, Copenhagen, Denmark

${ }^{93}$ Nikhef, National institute for subatomic physics, Amsterdam, Netherlands

${ }^{94}$ NRC Kurchatov Institute IHEP, Protvino, Russia

${ }^{95}$ NRC Kurchatov Institute-ITEP, Moscow, Russia

${ }^{96}$ NRNU Moscow Engineering Physics Institute, Moscow, Russia

${ }^{97}$ Nuclear Physics Group, STFC Daresbury Laboratory, Daresbury, United Kingdom

${ }^{98}$ Nuclear Physics Institute of the Czech Academy of Sciences, Řež u Prahy, Czech Republic

${ }^{99}$ Oak Ridge National Laboratory, Oak Ridge, Tennessee, USA

${ }^{100}$ Ohio State University, Columbus, Ohio, USA

${ }^{101}$ Petersburg Nuclear Physics Institute, Gatchina, Russia

${ }^{102}$ Physics department, Faculty of science, University of Zagreb, Zagreb, Croatia

${ }^{103}$ Physics Department, Panjab University, Chandigarh, India

${ }^{104}$ Physics Department, University of Jammu, Jammu, India

${ }^{105}$ Physics Department, University of Rajasthan, Jaipur, India

${ }^{106}$ Physikalisches Institut, Eberhard-Karls-Universität Tübingen, Tübingen, Germany

${ }^{107}$ Physikalisches Institut, Ruprecht-Karls-Universität Heidelberg, Heidelberg, Germany

${ }^{108}$ Physik Department, Technische Universität München, Munich, Germany

${ }^{109}$ Politecnico di Bari, and Sezione INFN, Bari, Italy

${ }^{110}$ Research Division and ExtreMe Matter Institute EMMI, GSI Helmholtzzentrum für Schwerionenforschung GmbH, Darmstadt, Germany

${ }^{111}$ Russian Federal Nuclear Center (VNIIEF), Sarov, Russia

${ }^{112}$ Saha Institute of Nuclear Physics, Homi Bhabha National Institute, Kolkata, India

${ }^{113}$ School of Physics and Astronomy, University of Birmingham, United Kingdom

${ }^{114}$ Sección Física, Departamento de Ciencias, Pontificia Universidad Católica del Perú, Lima, Peru

${ }^{115}$ St. Petersburg State University, St. Petersburg, Russia

${ }^{116}$ Stefan Meyer Institut für Subatomare Physik (SMI), Vienna, Austria

${ }^{117}$ SUBATECH, IMT Atlantique, Université de Nantes, CNRS-IN2P3, Nantes, France

${ }^{118}$ Suranaree University of Technology, Nakhon Ratchasima, Thailand

${ }^{119}$ Technical University of Košice, Košice, Slovakia

${ }^{120}$ The Henryk Niewodniczanski Institute of Nuclear Physics, Polish Academy of Sciences, Cracow, Poland

${ }^{121}$ The University of Texas at Austin, Austin, Texas, USA

${ }^{122}$ Universidad Autónoma de Sinaloa, Culiacán, Mexico

${ }^{123}$ Universidade de São Paulo (USP), São Paulo, Brazil

${ }^{124}$ Universidade Estadual de Campinas (UNICAMP), Campinas, Brazil

${ }^{125}$ Universidade Federal do ABC, Santo Andre, Brazil

${ }^{126}$ University of Cape Town, Cape Town, South Africa 


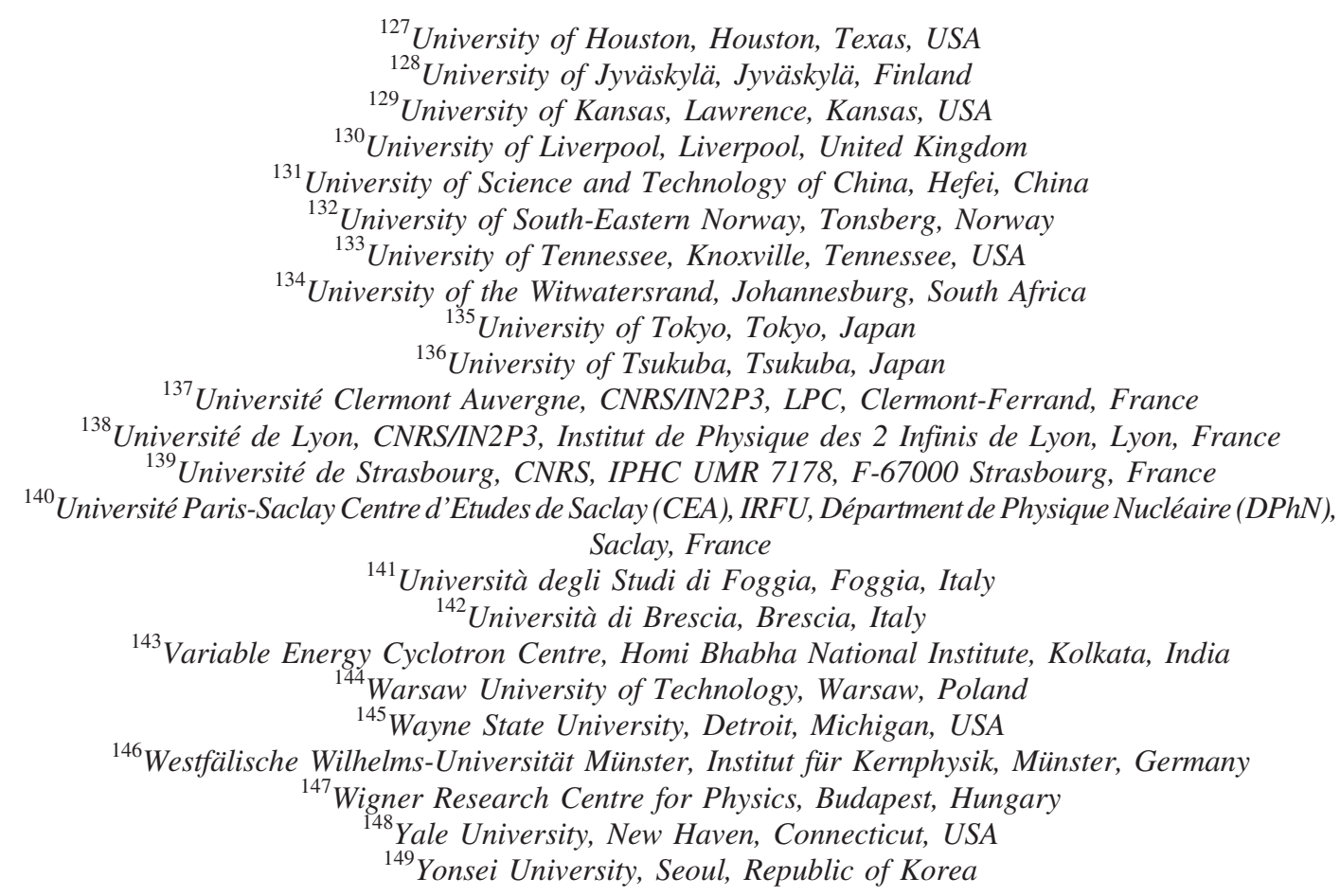

${ }^{\mathrm{a}}$ Deceased.

${ }^{\mathrm{b}}$ Also at Italian National Agency for New Technologies, Energy and Sustainable Economic Development (ENEA), Bologna, Italy. ${ }^{\mathrm{c}}$ Also at Dipartimento DET del Politecnico di Torino, Turin, Italy.

${ }^{\mathrm{d}}$ Also at M.V. Lomonosov Moscow State University, D.V. Skobeltsyn Institute of Nuclear, Physics, Moscow, Russia.

${ }^{\mathrm{e}}$ Also at Institute of Theoretical Physics, University of Wroclaw, Poland. 